\title{
An integrated model of soil-canopy spectral radiances, photosynthesis, fluorescence, temperature and energy balance
}

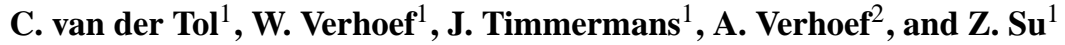 \\ ${ }^{1}$ ITC International Institute for Geo-Information Science and Earth Observations, Hengelosestraat 99, P.O. Box 6, 7500 AA, \\ Enschede, The Netherlands \\ ${ }^{2}$ Department of Soil Science, The University of Reading, Whiteknights, Reading, RG6 6DW, UK
}

Received: 20 May 2009 - Published in Biogeosciences Discuss.: 23 June 2009

Revised: 8 November 2009 - Accepted: 25 November 2009 - Published: 18 December 2009

\begin{abstract}
This paper presents the model SCOPE (Soil Canopy Observation, Photochemistry and Energy fluxes), which is a vertical (1-D) integrated radiative transfer and energy balance model. The model links visible to thermal infrared radiance spectra $(0.4$ to $50 \mu \mathrm{m})$ as observed above the canopy to the fluxes of water, heat and carbon dioxide, as a function of vegetation structure, and the vertical profiles of temperature. Output of the model is the spectrum of outgoing radiation in the viewing direction and the turbulent heat fluxes, photosynthesis and chlorophyll fluorescence. A special routine is dedicated to the calculation of photosynthesis rate and chlorophyll fluorescence at the leaf level as a function of net radiation and leaf temperature. The fluorescence contributions from individual leaves are integrated over the canopy layer to calculate top-of-canopy fluorescence. The calculation of radiative transfer and the energy balance is fully integrated, allowing for feedback between leaf temperatures, leaf chlorophyll fluorescence and radiative fluxes. Leaf temperatures are calculated on the basis of energy balance closure. Model simulations were evaluated against observations reported in the literature and against data collected during field campaigns. These evaluations showed that SCOPE is able to reproduce realistic radiance spectra, directional radiance and energy balance fluxes. The model may be applied for the design of algorithms for the retrieval of evapotranspiration from optical and thermal earth observation data, for validation of existing methods to monitor vegetation functioning, to help interpret canopy fluorescence measurements, and to study the relationships between synoptic observations with diurnally integrated quantities. The model has been implemented in Matlab and has a modular design, thus allowing for great flexibility and scalability.
\end{abstract}

Correspondence to: C. van der Tol (tol@itc.nl)

\section{Introduction}

Knowledge of physical processes at the land surface is relevant for a wide range of applications including weather and climate prediction, agriculture, and ecological and hydrological studies. Of particular importance are the fluxes of energy, carbon dioxide and water vapour between land and atmosphere.

During the last decades scientific understanding of physical processes at the land surface has grown, as a result of the increased availability of data, both from ground based and remote sensors. The implementation of a network of flux towers (FLUXNET) has increased the knowledge about processes at plot level in different ecosystems and different climates (Baldocchi, 2003). The knowledge from FLUXNET and earlier tower experiments has been widely incorporated in detailed coupled models for energy, carbon dioxide and water transport between soil, vegetation and atmosphere (e.g. Sellers et al., 1997; Verhoef and Allen, 2000; Tuzet et al., 2003).

Data from high resolution optical imagers, multi-spectral radiometers and radar on satellite platforms are nowadays available to retrieve spatial information about topography, soil and vegetation (CEOS, 2008). For example, Verhoef and Bach (2003) derived vegetation parameters by inverting a radiative transfer model on satellite derived hyperspectral reflectance data. Attempts have also been made to estimate evaporation from thermal images (Bastiaanssen, 1998). Furthermore, remote sensing (RS) data have been used as input for spatial soil-vegetation-atmosphere-transfer (SVAT) models for estimation of the surface energy balance (Kustas et al., 1994; Su, 2002; Anderson et al., 2008).

The potential of remote sensors operating at different spatial, temporal and spectral resolution is not yet fully exploited, for various reasons. First, remote sensing data is often of too coarse spatial resolution for SVAT models, which

Published by Copernicus Publications on behalf of the European Geosciences Union. 
are detailed and require field-scale data (Hall et al., 1992). Second, the relation between radiative transfer parameters and SVAT parameters is often indirect, due to their different scholarly background. For this reason it is not possible to directly translate, for example, an optical leaf area index which was successfully retrieved from a radiative transfer model into a one-sided leaf area index that is used in a photosynthesis model (Norman and Becker, 1995).

In order to make effective use of the available RS data, coherent models are needed for the interpretation of observed radiance spectra with respect to physical processes on the ground. These models should incorporate fluxes of water, carbon and energy at the land surface, as well as radiative transfer. The model of Goudriaan (1977) and the CUPID model (Norman, 1979; Kustas et al., 2007), to our knowledge, have been the first models for both radiative transfer and heat, water (vapour) and $\mathrm{CO}_{2}$ exchange in canopies. With these models, brightness temperature can be calculated for multiple-source canopies where leaves and soil have different temperatures. The models calculate radiation and energy fluxes in forward mode, which means that for the interpretation of observed spectra, they have to be inverted.

This paper presents a new model; SCOPE (Soil Canopy Observation of Photochemistry and Energy fluxes), which is a vertical (1-D) integrated radiative transfer and energy balance model. It calculates the spectral radiation regime and the energy balance of a vegetated surface at the level of single leaves as well as at canopy level, and the spectrum of the outgoing radiation in the viewing direction at a high spectral resolution over the range from 0.4 to $50 \mu \mathrm{m}$, thus including the visible, near and shortwave infrared, as well as the thermal domain. The spectral resolution in these regions can easily be adapted to simulation requirements and spectral input data, and is typically of the order of $10 \mathrm{~nm}$. The model calculates the energy balance of the surface (unlike CUPID, a site water balance is not calculated at present, thus requiring direct information on soil water content). The model also includes the computation of chlorophyll fluorescence and leaf and canopy level, which is relevant for investigations in the framework of the FLuorescence EXplorer (FLEX) mission of the ESA (Rascher et al., 2008).

Radiative transfer is described on the basis of the fourstream SAIL extinction and scattering coefficients (Verhoef, 1984), but the solution method of SCOPE is of a more numerical nature to allow for a heterogeneous vertical temperature distribution. The model shares its abstraction of canopy structure with the one applied in the FluorSAIL model (Miller et al., 2005). Regarding the modelling of thermal fluxes, in SCOPE the sunlit leaves are discriminated by their orientation with respect to the sun and their vertical position in the canopy layer, whereas in 4SAIL (Verhoef et al., 2007) only a differentiation between sunlit and shaded leaves was made.
The purpose of SCOPE is to facilitate better use of remote sensing data in modelling of water, carbon dioxide and energy fluxes at the land surface. SCOPE can support the interpretation of earth observation data in meteorological, hydrological, agricultural and ecological applications. The calculation of a broad electromagnetic spectrum ( 0.4 to $50 \mu \mathrm{m})$ allows for the simultaneous use of different sensors to bridge the observational spectral gaps. The model can be used at the plot scale as a theoretical 'ground truth' for testing simpler models, and as such to evaluate relationships between surface characteristics and (parts of) the reflected spectral radiation, such as the relation between indices (e.g. NDVI) and other vegetation characteristics (e.g. LAI). Because it is a 1$\mathrm{D}$ vertical model which assumes homogeneity in horizontal direction, the model may not be applicable for heterogeneous areas.

The aim of this paper is to describe the model structure and technical and implementation aspects. In this paper the model is described (Sect. 2), the output of the model is presented including a validation of spectra and fluxes (Sect. 3), and potential applications of the model are discussed (Sect. 4). A full validation of the model against field experiments will be presented in a following paper.

\section{Model description}

\subsection{Model structure}

The model SCOPE is based on existing theory of radiative transfer, micrometeorology and plant physiology. The strength of the model is the way in which interactions between the different model components are modelled. Three unique features of the model make it particularly relevant for future applications:

1. the use of the model PROSPECT (Jacquemoud and Baret, 1990) for optical properties of leaves in combination with a photosynthesis model;

2. the calculation of heterogeneous canopy and soil temperatures in combination with the energy balance;

3. the calculation of chlorophyll fluorescence as a function of irradiance, canopy temperature and other environmental conditions (in previous models, chlorophyll fluorescence was only a function of irradiance).

The model consists of a structured cascade of separate modules. These modules can be used stand alone, or, as in the integrated model, they can be connected by exchanging input and output. Depending on the application, some modules can be left out or replaced by others.

Figure 1 shows schematically how the main modules interact. The model distinguishes between modules for radiative transfer (of incident light, and internally generated thermal 


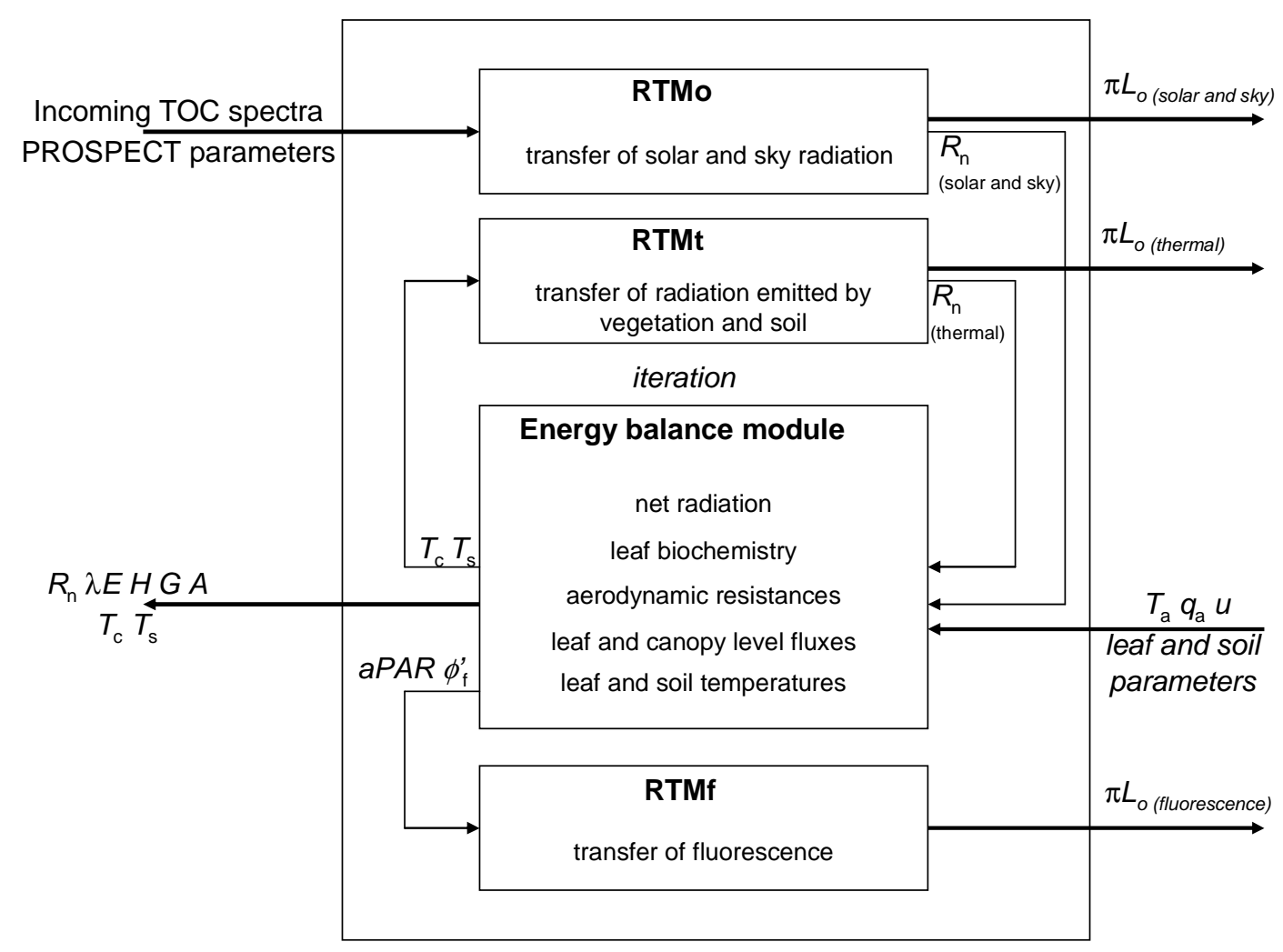

Fig. 1. Schematic overview of the SCOPE model structure.

radiation, and chlorophyll fluorescence), and the energy balance. The modules are executed in the order from top to bottom of the figure:

1. RTMo, a semi-analytical radiative transfer module for incident solar and sky radiation, based on SAIL (Verhoef and Bach, 2007): calculates the TOC (top of canopy) outgoing radiation spectrum $(0.4$ to $50 \mu \mathrm{m})$, as well as the net radiation and absorbed photosynthetically active radiation (PAR) per surface element.

2. RTMt, a numerical radiative transfer module for thermal radiation generated internally by soil and vegetation, based on Verhoef et al. (2007): calculates the TOC outgoing thermal radiation and net radiation per surface element, but for heterogeneous leaf and soil temperatures.

3. A new energy balance module for latent, sensible and soil heat flux per surface element, as well as photosynthesis, chlorophyll fluorescence and skin temperature at leaf level.

4. RTMf, a radiative transfer module for chlorophyll fluorescence based on the FluorSAIL model (Miller et al., 2005): calculates the TOC radiance spectrum of fluorescence from leaf level chlorophyll fluorescence (calculated in step 3) and the geometry of the canopy.
Iteration between the thermal radiative transfer module (RTMt) and the energy balance module is carried out to match the input of the radiative transfer model with the output of the energy balance model (skin temperatures), and vice versa: the input of the energy balance model with the output of the radiative transfer model (net radiation). For computational efficiency, the radiative transfer of chlorophyll fluorescence (RTMf) is carried out at the end of the cascade, which implies that the contribution of chlorophyll fluorescence to the energy balance is neglected. Its contribution to the outgoing radiance spectrum is finally added to the reflectance. Note that this only holds for the radiative transfer and the calculation of the TOC spectrum of chlorophyll fluorescence (RTMf), which is computationally demanding. The chlorophyll fluorescence at leaf level is calculated every iteration step as a by-product of the photosynthesis model (step 3).

The radiative transfer modules serve two purposes: first, to predict the TOC radiance spectrum in the observation direction, and second, to predict the distribution of irradiance and net radiation over surface elements (leaves and the soil). The latter is input for the energy balance module. The energy balance module serves two purposes as well: first to calculate the fate of net radiation (i.e. the turbulent energy fluxes and photosynthesis), and secondly to calculate surface temperature and fluorescence of the elements of the surface. The latter are input for the radiative transfer model. Sharing 
input, output and parameters makes it possible to study the relationship between TOC spectra and energy fluxes in a consistent way. For example, the energy balance is preserved at all times (except for the small contribution of chlorophyll fluorescence).

For the calculation of radiative transfer, the description of the geometry of the vegetation is of crucial importance. Leaves and soil are divided into classes which receive a similar irradiance. These classes are the elements of the model. This distinction of elements is a stochastic technique to describe the effects of the geometry of the vegetation on the outgoing spectrum and on the heterogeneity of net radiation.

The geometry of the canopy is described as follows. It is assumed that the canopy has a homogeneous structure, and is 1-D only, which means that variations of macroscopic properties in the horizontal plane as well as clumping of twigs and branches are neglected. For the purpose of numerical radiative transfer calculations, we define 60 elementary layers, with a maximum LAI of 0.1 each, so that numerical approximations to the radiative transfer equations are still acceptable up to a total canopy LAI of 6. For the description of canopy architecture, the same as the one used in the SAIL models (Verhoef, 1984, 1998) is applied, which requires a total LAI, two parameters describing the leaf angle distribution and the hot spot parameter. Numerically, 13 discrete leaf inclinations are used as in SAIL, and the uniform leaf azimuth distribution is now also discretised to 36 angles of $5,15, \ldots, 355^{\circ}$ relative to solar azimuth. The numbers of elementary layers, leaf inclinations and leaf azimuth angles are actually variables, but these were set fixed at the given values to facilitate comparison with earlier models like FluorSAIL. This is especially useful for verification of the model's functioning in an early stage.

The elements of the model are defined as follows. For shaded leaves, 60 elements are distinguished (corresponding to the 60 leaf layers), since for the assumed semi-isotropic diffuse incident fluxes the leaf orientation is immaterial for the amount of flux that is intercepted. For sunlit leaves, $60 \times 13 \times 36$ elements (60 leaf layers, 13 leaf inclinations, $\theta_{\ell}$, and 36 leaf azimuth angles, $\varphi_{\ell}$ ) are distinguished, since the interception of solar flux depends on the orientation of the leaf with respect to the sun. The soil is divided into two elements: a shaded and a sunlit fraction.

In the model, the principle of linearity of the radiative transfer equation is exploited by combining the solutions for various standard boundary conditions and source functions, such as the ones related to the optical domain, the thermal domain, and the ones related to direct solar radiation, sky radiation, leaves in the sun, and leaves in the shade. The latter distinction is particularly important for the biochemistry components of the model (photosynthesis and fluorescence). Calculations for different parts of the spectrum, sources of radiation, and elements of the surface are carried out separately, and total fluxes are obtained afterwards by adding the different contributions. This makes it possible to separate the calculation of chlorophyll fluorescence, optical and thermal radiation and the calculation of different components of the surface, without violating energy conservation. This principle is exploited at several places in the model to enhance the computational efficiency and to create a transparent code.

In the following sections, the model is described in more detail. The modules are presented in an order which facilitates the conceptual understanding of the model, which is with very few exceptions also the order in which they are executed by the model (Fig. 1). We shall start with a description of the input at the top of the canopy (Sect. 2.2), followed by the radiative transfer models (Sect. 2.3 and 2.4), the calculation of net radiation (Sect. 2.5), the energy balance (Sect. 2.6), leaf biochemical processes (Sect. 2.7), and top-of -canopy outgoing radiance (Sect. 2.8).

\subsection{Atmospheric optical inputs}

The model SCOPE requires top-of-canopy incident radiation as input, at a spectral resolution high enough to take the atmospheric absorption bands properly into account. For the top of the canopy the incident fluxes from the sun and the sky can be obtained from the atmospheric radiative transfer model MODTRAN (Berk et al., 2000). The calculation of TOC incident fluxes is ideally done with MODTRAN before each simulation with SCOPE, using the actual values of solar zenith and azimuth angle and atmospheric conditions. An alternative is to create a library of incoming spectra, from which SCOPE can extract a typical spectrum for specific conditions. In this study, only one example spectrum was created with MODTRAN4. The shape of this example spectrum is used throughout the paper, while the magnitudes of the optical and thermal part of the spectrum are each linearly scaled according to local broadband measurements of incident irradiance.

From MODTRAN the following outputs are needed:

TRAN $=$ direct transmittance from target to sensor,

SFEM $=$ radiance contribution due to thermal surface emission,

GSUN = ground-reflected radiance due to direct solar radiation,

GRFL $=$ total ground-reflected radiance contribution .

An important quantity for the interaction between surface and atmosphere is the spherical albedo for illumination from below, especially at the shorter wavelengths. Two MODTRAN4 runs, for surface albedos of $50 \%$ and $100 \%$, are sufficient to estimate the spherical albedo of the atmosphere and the diffuse and direct solar fluxes incident at the top of the canopy. These MODTRAN runs should be done for a low sensor height ( $1 \mathrm{~m}$ above the surface is recommended) under nadir viewing angle, in order to keep the atmospheric transmittance from target to sensor as high as possible. With numerical subscripts indicating the surface albedo percentage, 
all relevant atmospheric and surface quantities can be determined as follows:

$$
\begin{aligned}
& \rho_{d d}=\frac{\mathrm{GRFL}_{100}-2 \times \mathrm{GRFL}_{50}}{\mathrm{GRFL}_{100}-\mathrm{GRFL}_{50}-\mathrm{SFEM}_{50}}, \\
& \tau_{o o}=\mathrm{TRAN}, \\
& L_{s}=2 \times \mathrm{SFEM}_{50} / \mathrm{TRAN}, \\
& O+T=\left(1-\rho_{d d}\right)\left(\mathrm{GRFL}_{100}-2 \times \mathrm{SFEM}_{50}\right) / \mathrm{TRAN}, \\
& E_{\text {sun }}=\pi \times \mathrm{GSUN}_{100} / \mathrm{TRAN} .
\end{aligned}
$$

Here, $\rho_{d d}$ is the spherical albedo of the atmosphere for illumination from below, $\tau_{o o}$ is the direct transmittance (TRAN) from ground to sensor. Note, that TRAN has no numerical subscript since it is independent of the surface albedo. The double subscripts appended to optical properties like $\rho$ (reflectance) and $\tau$ (transmittance) indicate the types of ingoing and outgoing fluxes, where $s$ stands for direct solar flux, $d$ for upward or downward diffuse flux and $o$ for flux (radiance) in the observation direction (Verhoef and Bach, 2003). See also the list of symbols, Table 1. Other symbols in Eqs. (1)-(5) are $L_{s}$, the blackbody surface radiance due to thermal emission, $E_{\text {sun }}$, the solar irradiance on the horizontal ground surface (or the top of the canopy), and the term $O+T$, which stands for a certain combination of optical and thermal quantities that is independent of the surface albedo. The importance of this term is related to the fact that it can be derived from the MODTRAN outputs and it is required for the estimation of the sky irradiance.

The sky irradiance onto the surface, $E_{\text {sky, }}$, is a derived quantity, which depends partly on the surface albedo in the surroundings. For arbitrary atmospheric conditions it can be estimated by

$E_{\mathrm{sky}}=\pi\left[\frac{O+T}{1-r_{s} \rho_{d d}}+L_{s}\right]-E_{\mathrm{sun}}$,

where $r_{s}$ is the surface albedo,

$$
\begin{aligned}
& O=\left(\tau_{s s}+\tau_{s d}\right) E_{s}(\mathrm{t}) / \pi \\
& T=L_{a}(\mathrm{~b})-\left(1-\rho_{d d}\right) L_{s},
\end{aligned}
$$

and where $E_{S}(\mathrm{t})$ is the extraterrestrial (TOA) solar irradiance on a plane parallel to the horizontal plane at ground level, $L_{a}(\mathrm{~b})$ is the thermal emitted sky radiance at the bottom of the atmosphere (BOA), assumed to be isotropic. Note that $(\mathrm{t})$ and (b) indicate the top and the bottom of the atmosphere, respectively. The transmittances $\tau_{s s}$ and $\tau_{s d}$ are the direct and the diffuse transmittances for direct solar flux from TOA to the ground.

As an example, and to illustrate the broad spectral range involved, Fig. 2 shows the spectra of $E_{\text {sun }}$ and $E_{\text {sky }}$ (in $\mathrm{W} \mathrm{m}{ }^{-2} \mu \mathrm{m}^{-1}$ ) for a surface albedo of zero. From these results it can be concluded that at $2.5 \mu \mathrm{m}$ already the diffuse sky irradiance starts to rise due to thermal emission, and at

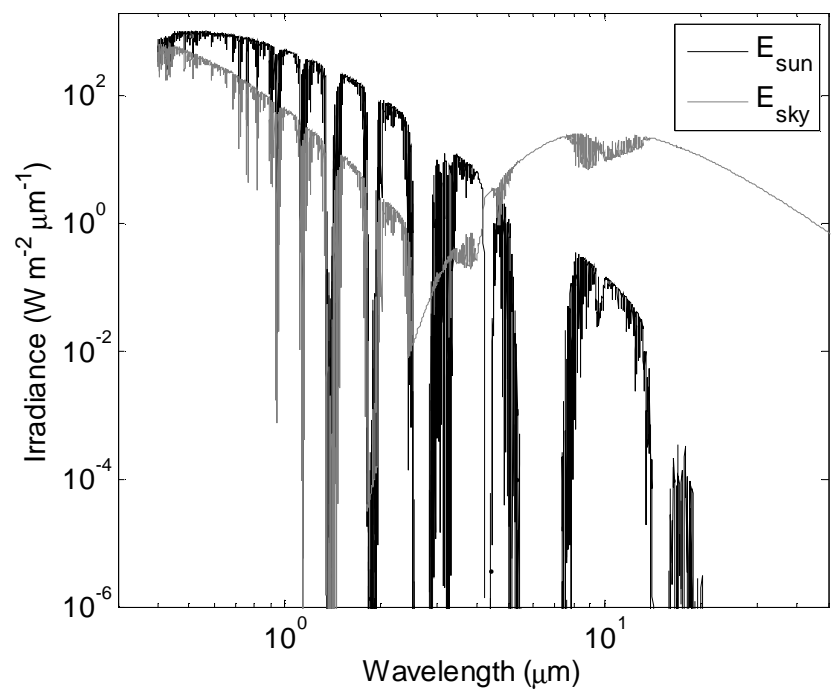

Fig. 2. Downward direct $\left(E_{\text {sun }}\right)$ and diffuse $\left(E_{\text {sky }}\right)$ irradiances (for zero albedo) on logarithmic scales. Plotted wavelength range is 0.4 to $50 \mu \mathrm{m}$. The solar zenith angle is $30^{\circ}$.

wavelengths longer than $8 \mu \mathrm{m}$ it is the dominant source of incident radiation. In spectral regions of low atmospheric absorption (high transmittance) the thermal sky radiance is less than in absorption bands. This is caused by the correspondingly lower atmospheric emissivity and the fact that higher and thus colder layers of the atmosphere contribute to the radiance at surface level.

Note that Eqs. (5) and (6) are used to calculate the atmospheric spectral inputs of SCOPE, but they are not part of the model code itself.

\subsection{Direct and diffuse radiation fluxes}

In the first radiative transfer module of SCOPE, the effects of thermal emission by surface elements are ignored and in this case the analytical solutions for the diffuse and direct fluxes as obtained from the SAIL model are used to calculate the vertical profiles of these fluxes inside the canopy layer. In addition, net radiation and absorbed PAR are calculated for soil and leaf elements.

For the diffuse upward $\left(E^{+}\right)$and downward $\left(E^{-}\right)$fluxes ( $\mathrm{W} \mathrm{m}^{-2} \mu \mathrm{m}^{-1}$ ), use is made of numerically stable analytical solutions as provided in the more recent 4SAIL model (Verhoef et al., 2007). This is further explained in Appendix A. The direct solar flux is described by

$E_{S}(x)=E_{S}(0) P_{S}(x)$,

where $E_{S}(0)$ is the direct solar flux incident at the top of the canopy $\left(E_{\mathrm{sun}}\right)$, and $P_{S}(x)$ is the probability of leaves or soil being sunlit (or the gap fraction in the solar direction), which is given by $P_{S}(x)=\exp (-k L x)$, where $x$ is the relative optical height $([-1,0]$, where -1 is at the soil surface and 0 at TOC), $L$ is the leaf area index (LAI), and $k$ is the 
Table 1. List of symbols.

\begin{tabular}{|c|c|c|}
\hline Symbol & Description & Unit \\
\hline$a$ & Attenuation coefficient & \\
\hline$A$ & Gross photosynthesis rate & $\mu \mathrm{mol} \mathrm{m}{ }^{-2} \mathrm{~s}^{-1}$ \\
\hline$b$ & Index for bottom of canopy & \\
\hline$c_{d}$ & Drag coefficient & \\
\hline$c_{p}$ & Heat capacity of the air & $\mathrm{J} \mathrm{kg}^{-1} \mathrm{~K}^{-1}$ \\
\hline$d$ & Zero-plane displacement height & $\mathrm{m}$ \\
\hline$E^{-}, E^{+}$ & Downward and upward irradiance & $\mathrm{W} \mathrm{m} \mathrm{m}^{-2} \mu \mathrm{m}^{-1}$ \\
\hline$E_{a p}$ & Absorbed photosynthetically active radiation & $\mu \mathrm{mol} \mathrm{m}^{-2} \mathrm{~s}^{-1}$ \\
\hline$E_{S}$ & Direct solar irradiance in the canopy & $\mathrm{W} \mathrm{m}^{-2} \mu \mathrm{m}^{-1}$ \\
\hline$E_{\text {sun }}, E_{\text {sky }}$ & Solar and sky irradiance above the canopy & $\mathrm{W} \mathrm{m}^{-2} \mu \mathrm{m}^{-1}$ \\
\hline$F_{1}, F_{2}$ & Transformed fluxes & $\mathrm{W} \mathrm{m}^{-2} \mu \mathrm{m}^{-1}$ \\
\hline$f_{o}, f_{s}$ & Leaf area projection factors in the directions of view and the sun & \\
\hline$f\left(\theta_{\ell}\right)$ & Leaf inclination distribution function & \\
\hline$G$ & Soil heat flux & $\mathrm{W} \mathrm{m}^{-2}$ \\
\hline$h$ & Vegetation height & $\mathrm{m}$ \\
\hline$H$ & Sensible heat flux & $\mathrm{W} \mathrm{m}^{-2}$ \\
\hline$H_{C}, H_{S}$ & Blackbody emission by vegetation and soil & $\mathrm{W} \mathrm{m}^{-2}$ \\
\hline$J_{1}, J_{2}$ & Functions to establish numerically stable solutions in SAIL & \\
\hline$J_{\max }$ & Maximum electron transport capacity & $\mu \mathrm{mol} \mathrm{m}^{-2} \mathrm{~s}^{-1}$ \\
\hline$k$ & Extinction coefficient in solar direction & \\
\hline$K$ & Extinction coefficient in observation direction & \\
\hline$K_{h, v}$ & Eddy diffusivity & $\mathrm{m}^{2} \mathrm{~s}^{-1}$ \\
\hline$K_{r}$ & Von Kármán’s constant & \\
\hline$\ell$ & Ratio of leaf width to canopy height & \\
\hline$L$ & Leaf area index (always without a subscript) & \\
\hline$L$ & Spectral radiance (always to be used with subscripts) & $\mathrm{W} \mathrm{m}^{-2} \mu \mathrm{m}^{-1} \mathrm{sr}^{-1}$ \\
\hline$m$ & Eigenvalue of two-stream diffuse radiative transfer equation & \\
\hline$M, M^{\prime}$ & Backward and forward fluorescence matrix & \\
\hline$n$ & Wind extinction coefficient & \\
\hline$P_{s}, P_{o}, P_{s o}$ & Gap fractions for sunshine, observation, and observation of sunlit elements & \\
\hline$q$ & Generic extinction coefficient (can be $K$ or $k$ ) & \\
\hline$q_{s}, q_{a}$ & Absolute humidity of the surface and the air & $\mathrm{kg} \mathrm{m}^{-3}$ \\
\hline$r_{\infty}$ & Bi-hemispherical canopy reflectance for infinite LAI & \\
\hline$r_{a}, r_{c}, r_{w}, r_{b}$ & Aerodynamic and surface resistance, within vegetation and boundary resistance & $\mathrm{s} \mathrm{m}^{-1}$ \\
\hline$R_{d}$ & Dark respiration rate & $\mu \mathrm{mol} \mathrm{m}{ }^{-2} \mathrm{~s}^{-1}$ \\
\hline$R_{n}$ & Net radiation & $\mathrm{W} \mathrm{m}^{-2}$ \\
\hline$r_{s}$ & Soil or surface reflectance & \\
\hline$r_{s o}, r_{d o}$ & Canopy-level reflectances for direct and diffuse radiation in observation direction & \\
\hline$s, s$ & Backscatter and forward scatter coefficient for solar incident flux in the canopy & \\
\hline$t$ & Time & $\mathrm{s}$ \\
\hline $\mathrm{t}$ & Index for top of canopy & \\
\hline$T_{a}, T_{c}, T_{s}$ & Air, vegetation and soil temperature & ${ }^{\circ} \mathrm{C}$ \\
\hline$u$ & Wind speed & $\mathrm{ms}^{-1}$ \\
\hline$u_{*}$ & Friction velocity & $\mathrm{ms}^{-1}$ \\
\hline$v, v^{\prime}$ & $\begin{array}{l}\text { Scattering coefficients for downward and upward diffuse fluxes } \\
\text { into observed radiance times } \pi\end{array}$ & \\
\hline$V_{\text {cmax }}$ & Maximum carboxylation rate & $\mu \mathrm{mol} \mathrm{m}^{-2} \mathrm{~s}^{-1}$ \\
\hline$V_{\text {pmo }}$ & Maximum PEP regeneration rate (for $\mathrm{C} 4$ crops only) & $\mu \mathrm{mol} \mathrm{m}^{-2} \mathrm{~s}^{-1}$ \\
\hline$w$ & Bi-directional scattering coefficient & \\
\hline$w_{l}$ & Leaf width & $\mathrm{m}$ \\
\hline$x$ & Relative depth in the canopy $[-1,0]$ & \\
\hline$z, z_{r}, z_{R}, z_{0 \mathrm{~m}}$ & $\begin{array}{l}\text { Measurement height, height of the inertial sublayer, height of the roughness sublayer, } \\
\text { roughness length for momentum }\end{array}$ & $\mathrm{m}$ \\
\hline$\alpha$ & Leaf absorptance & \\
\hline
\end{tabular}


Table 1. Continued.

\begin{tabular}{|c|c|c|}
\hline Symbol & Description & Unit \\
\hline$\Gamma$ & Thermal inertia of the soil & $\mathrm{JK}^{-1} \mathrm{~m}^{-2} \mathrm{~s}^{-1 / 2}$ \\
\hline$\delta_{o}$ & Angle between leaf surface normal and observation direction & \\
\hline$\delta_{S}$ & Angle between leaf surface normal and solar direction & \\
\hline$\delta_{1}, \delta_{2}$ & Boundary constants for diffuse fluxes & \\
\hline$\varepsilon_{c}, \varepsilon_{s}$ & Emissivity for vegetation and soil & \\
\hline$\theta_{\ell}$ & Leaf inclination angle $=$ leaf normal zenith angle & \\
\hline$\theta_{o}, \theta_{s}$ & Observation and solar zenith angles & \\
\hline$\kappa$ & Extinction coefficient for diffuse fluxes & \\
\hline$\lambda$ & Vaporization heat of water & $\mathrm{J} \mathrm{kg}^{-1}$ \\
\hline$\lambda_{c}$ & Cowan's water use efficiency parameter & \\
\hline$\lambda E$ & Latent heat & $\mathrm{W} \mathrm{m}^{-2}$ \\
\hline$\lambda_{e}, \lambda_{f}$ & Excitation and fluorescence wavelength & $\mu \mathrm{m}$ \\
\hline$\Lambda$ & Monin-Obukhov length & $\mathrm{m}$ \\
\hline$\rho$ & Leaf reflectance & \\
\hline$\rho_{a}$ & Air density & $\mathrm{kg} \mathrm{m}^{-3}$ \\
\hline$\rho_{s}$ & Soil reflectance & \\
\hline$\sigma, \sigma^{\prime}$ & Diffuse backscatter, forward scatter coefficient & \\
\hline$\tau$ & Leaf transmittance & \\
\hline$\Phi$ & Stability correction function (Eqs. B13 and B17) & \\
\hline$\phi_{f}^{\prime}$ & $\begin{array}{l}\text { Leaf fluorescence as a fraction of that in unstressed, low light conditions, } \\
\text { in energy units of incident PAR }\end{array}$ & \\
\hline$\varphi_{\ell}$ & Leaf azimuth angle (relative to solar azimuth) & \\
\hline$\varphi_{o}, \varphi_{s}$ & Observation and solar azimuth angles & \\
\hline$\Psi$ & Stability correction function (Eqs. B14-B16) & \\
\hline$\Psi$ & Azimuth angle of observation relative to solar azimuth & $\mathrm{rad}$ \\
\hline$\omega$ & Frequency of the diurnal cycle & $\operatorname{rads}^{-1}$ \\
\hline
\end{tabular}

extinction coefficient in the direction of the sun. As shown in Appendix A, the diffuse upward and downward fluxes are derived from transformed fluxes $F_{1}$ and $F_{2}$, which are given by

$$
\begin{aligned}
& F_{1}(x)=\delta_{1} e^{m L x}+\left(s^{\prime}+r_{\infty} s\right) E_{s}(0) J_{1}(k, x) \\
& F_{2}(x)=\delta_{2} e^{-m L(1+x)}+\left(r_{\infty} s^{\prime}+s\right) E_{s}(0) J_{2}(k, x)
\end{aligned}
$$

where $m$ is the eigenvalue of the diffuse flux system, $r_{\infty}$ is the infinite reflectance (i.e. the bi-hemispherical reflectance for infinite LAI), $s$ the backscatter coefficient, $s^{\prime}$ the forward scatter coefficient, $J_{1}$ and $J_{2}$ numerically stable functions as described in Verhoef and Bach (2007), and $\delta_{1}$ and $\delta_{2}$ are boundary constants. In Appendix A, more extensive information is given about the SAIL coefficients, their use in the analytical solution, the boundary constants for given solar and sky irradiance, and the incorporation of the soil's reflectance.

The coefficients $m, s, s^{\prime}$ and $r_{\infty}$ in Eq. (9) depend on the transmittance and reflectance of the leaves and the leaf inclination distribution. The spectral transmittance and reflectance of the leaves are calculated with the model PROSPECT (Jacquemoud and Baret, 1990), using the concentrations of leaf water, chlorophyll, dry matter, and brown pigment, as well as the leaf mesophyll scattering parameter $N$, as input parameters. The soil's reflectance spectrum is another required input. In this study, a standard spectrum for a loamy sand soil was used.

\subsection{Internally generated thermal radiation}

The incident radiation on leaves should not only include the optical and thermal radiation from sun and sky, but also all thermal radiation that is generated internally by leaves and by the soil. In Verhoef et al. (2007) the thermal domain was treated by means of an analytical solution, which assumed distinct, but otherwise constant, temperatures of sunlit and shaded leaves, as well as sunlit and shaded soil. However, one may expect that in reality all leaves will all have different temperatures, depending on their orientation with respect to the sun, and their vertical position in the canopy layer (Timmermans et al., 2008). Therefore, a numerical solution allowing more temperature variation is preferred. For this, the energy balance equation is solved at the level of individual leaves, for $13 \times 36$ leaf orientations (leaf inclination $\theta_{\ell}$ and leaf azimuth $\varphi_{\ell}$ ), and 60 vertical positions in the canopy layer.

In order to compute the internally generated fluxes by thermal emission from leaves and the soil, it is initially assumed that the temperature of the leaves and the soil are equal to the air temperature. Next, the external radiation sources are 
added, and the energy balance is solved. This gives new temperatures of the leaves and the soil, whereby also sunlit and shaded components are distinguished.

For the numerical solution of this problem, we start with the two-stream differential equations in which absorption, scattering and thermal emission are included. These are given by

$$
\begin{aligned}
\frac{\mathrm{d}}{L \mathrm{~d} x} E^{-} & =a E^{-}-\sigma E^{+}-\varepsilon_{c} H_{c} \\
\frac{\mathrm{d}}{L \mathrm{~d} x} E^{+} & =\sigma E^{-}-a E^{+}+\varepsilon_{c} H_{c}
\end{aligned}
$$

where $a$ is the attenuation coefficient, $\sigma$ the backscatter coefficient, $\varepsilon_{c}$ the emissivity of the leaves (canopy), and $H_{c}$ the black body emittance. The attenuation coefficient is the diffuse extinction coefficient $\kappa$ minus the forward scattering coefficient $\sigma^{\prime}$, so $a=\kappa-\sigma^{\prime}$. This is because forward scattered radiation does not contribute to net attenuation. The blackbody emittance of the leaves is given by $H_{c}=\pi B\left(T_{c}\right)$, where $T_{c}$ is the vegetation's skin temperature, and $B$ the Planck blackbody radiance function. Alternatively, radiation integrated over the spectrum can be calculated by using StefanBoltzmann's equation for black body radiation.

The emitted radiation fluxes are calculated at the level of single leaves. In order to model $E^{-}$and $E^{+}$on the basis of Eq. (10), one needs the emitted radiation at the level of leaf layers. The layer-level fluxes are calculated by applying a weighted averaging, taking into account the leaf inclination distribution, $f\left(\theta_{\ell}\right)$, and the probability of sunshine, $P_{s}$, in order to differentiate between leaves in the sun (subscript "s") and leaves in the shade (subscript " $d$ "):

$$
\begin{aligned}
& H_{c}(x) \\
& =P_{s}(x) \sum_{\substack{13 \theta_{\ell} \\
36 \varphi_{\ell}}} f\left(\theta_{\ell}\right) H_{c s}\left(x, \theta_{\ell}, \varphi_{\ell}\right) / 36+\left[1-P_{s}(x)\right] H_{c d}(x) .
\end{aligned}
$$

The emittances $H_{c s}$ and $H_{c d}$ are the thermal emitted fluxes from individual leaves in the sun and in the shade, respectively. In order to numerically solve Eq. (10), we use the corresponding differential equations for the transformed diffuse fluxes, $F_{1}=E^{-}-r_{\infty} E^{+}$and $F_{2}=-r_{\infty} E^{-}+E^{+}$, where $r_{\infty}=(a-m) / \sigma$. It can be shown that the associated differential equations for the transformed fluxes are given by:

$$
\begin{gathered}
\frac{\mathrm{d}}{L \mathrm{~d} x} F_{1}=m F_{1}-m\left(1-r_{\infty}\right) H_{c} \\
\frac{\mathrm{d} x}{L \mathrm{~d} x} F_{2}=-m F_{2}+m\left(1-r_{\infty}\right) H_{c}
\end{gathered}
$$

where $m=\sqrt{\left(a^{2}-\sigma^{2}\right)}$ is the eigenvalue of the diffuse flux system. These differential equations have the advantage that (if thermal emission were absent) vertical propagation of the transformed fluxes would be simplified to purely exponential decays in downward and upward directions and that only one independent variable is involved at a time, which leads to a quick convergence.
For the fluxes at the soil level one can write

$E^{+}(-1)=r_{s} E^{-}(-1)+\left(1-r_{s}\right) H_{s}$,

where $r_{s}$ is the soil's reflectance and $H_{s}$ is the black body emittance of the soil.

Soil emitted radiation is calculated as a weighted sum of sunlit and shaded soil:

$H_{s}=P_{s}(-1) H_{s s}+\left[1-P_{s}(-1)\right] H_{s d}$.

Equations (12) and (13) can be used as the basis for a numerical solution of the fluxes in the case of heterogeneous foliage temperatures. An advantage of working with transformed fluxes is that these can be directly expressed in those of the layer above or below the current one, and for a finite difference numerical solution we obtain the simple recursive equations

$$
\begin{aligned}
& F_{1}(x-\Delta x)=(1-m L \Delta x) F_{1}(x)+m\left(1-r_{\infty}\right) H_{c}(x) L \Delta x, \\
& F_{2}(x+\Delta x)=(1-m L \Delta x) F_{2}(x)+m\left(1-r_{\infty}\right) H_{c}(x) L \Delta x .
\end{aligned}
$$

If the first transformed flux is given at the canopy top, it can be propagated downwards to the soil level. Next, the second transformed flux can be started at the soil level, and it can be propagated upwards to the top-of-canopy (TOC) level. However, the second transformed flux at the soil level is not known initially, so it has to be derived from the boundary equation, Eq. (13). This gives

$$
F_{2}(-1)=\frac{\left(r_{s}-r_{\infty}\right)}{\left(1-r_{s} r_{\infty}\right)} F_{1}(-1)+\frac{\left(1-r_{\infty}^{2}\right)}{\left(1-r_{s} r_{\infty}\right)}\left(1-r_{s}\right) H_{s}
$$

This relation can be used to link the downward and upward sequences of the difference Eqs. (15), and finally both transformed fluxes at the TOC level will be available.

The initial guess of $F_{1}(0)$ is made under the assumption that there is no downward incident flux at the top of the canopy. Since in the thermal infrared $r_{\infty}$ is also small, we simply assume that initially $F_{1}(0)$ is zero. Note that only thermal radiation emitted by leaves and soil is considered here. Thermal radiation from the sky, which is not negligible, has been treated with the semi-analytical solution described in Sect. 2.3.

After the downward and upward sequences have been completed, also the second transformed flux at TOC level, $F_{2}(0)$, is known. At this moment one can correct the initial guess of $F_{1}(0)$, since it was based on the assumption of an upward flux of zero. For this, use is made of the equation

$F_{1}(0)+r_{\infty} F_{2}(0)=\left(1-r_{\infty}^{2}\right) E^{-}(0)=0$,

which is rewritten as $F_{1}(0)=-r_{\infty} F_{2}(0)$. 
Summarized, the algorithm works as follows:

1. Assume $F_{1}(0)=0$.

2. Propagate Eq. (15a) down to the soil level, giving $F_{1}(-1)$.

3. Apply Eq. (16), giving $F_{2}(-1)$.

4. Propagate Eq. (15b) up to TOC level, giving $F_{2}(0)$.

5. Apply $F_{1}(0)=-r_{\infty} F_{2}(0)$, and go back to step 2, unless the change is less than a given threshold.

In practice, a couple of iterations are usually sufficient to arrive at the correct fluxes at both boundaries.

The emissivity parameters $\varepsilon_{c}$ and $\varepsilon_{s}$ are input of the model. In this study, uniform a priori values over the thermal spectrum were used. In future versions of the model, more knowledge about the spectral distribution of emissivity in the thermal range might be incorporated.

\subsection{Net radiation}

Net radiation includes the contributions of all radiation from 0.4 to $50 \mu \mathrm{m}$. Here, the principle of linearity of the fluxes is exploited to integrate the energy fluxes, over the spectrum, over the source of radiation and over elements. This implies that the solution obtained from the semi-analytical module for solar and sky radiation (Sect. 2.3) and the solution for internally generated thermal radiation (Sect. 2.4) can be added. Net radiation of a layer is the weighted sum of the contributions from shaded and sunlit leaves with different leaf angles. Similarly, net radiation of the canopy is the sum of the contributions of the individual layers.

The net spectral radiation on a leaf is equal to the absorption minus its total emission from the two sides, or, for leaves in the shade

$R_{n}(x)=\left[E^{-}(x)+E^{+}(x)-2 H_{c d}(x)\right](1-\rho-\tau)$.

In this equation, $E^{-}$and $E^{+}$are the sum of the externally (solar and sky) and internally generated fluxes. It is assumed that leaf emissivity $\varepsilon$ equals leaf absorptance $\alpha=1-\rho-\tau$ (Kirchhoff's Law), where $\rho$ and $\tau$ are the reflectance and the transmittance of the leaf. For leaves in the sun with a given orientation relative to the sun we obtain

$$
\begin{aligned}
& R_{n}\left(x, \theta_{\ell}, \varphi_{\ell}\right)= \\
& {\left[\left|f_{s}\right| E_{\text {sun }}+E^{-}(x)+E^{+}(x)-2 H_{c s}\left(x, \theta_{\ell}, \varphi_{\ell}\right)\right](1-\rho-\tau) .}
\end{aligned}
$$

Here,

$f_{s}=\frac{\cos \delta_{s}}{\cos \theta_{s}}=\frac{\cos \theta_{s} \cos \theta_{\ell}+\sin \theta_{s} \sin \theta_{\ell} \cos \varphi_{\ell}}{\cos \theta_{s}}$,

where $\theta_{s}$ is the solar zenith angle and the leaf azimuth $\varphi_{\ell}$ is taken to be relative with respect to the solar azimuth.
The numerator of the above expression is the projection of the leaf onto a plane perpendicular to the sunrays. Its absolute value is maximal if the leaf's normal points to the sun or in the opposite direction. The division by the cosine of the solar zenith angle is applied because the solar irradiance is also defined for a horizontal plane. If the leaf's normal points to the sun, it receives more radiation than a horizontal surface would. The leaf's emittances (emitted fluxes) are defined for leaves in the shade and in the sun. For leaves in the shade the emittance depends only on the vertical position. Leaves in the sun will all have different temperatures and emittances, depending on their orientation and vertical position (Sect. 2.4).

\subsection{The energy balance}

The fate of net radiation is calculated per element with the energy balance model. The energy balance model distributes net radiation over turbulent air fluxes and heat storage.

The energy balance equation for each element $i$ is given by:

$R_{n}-H-\lambda E-G=0$,

where $R_{n}$ is net radiation, $H$ is sensible and $\lambda E$ is latent heat flux, whereas $G$ is the change in heat storage (all in $\mathrm{W} \mathrm{m}^{-2}$ ). In this equation, energy involved in the melting of snow and freezing of water is not considered, and energy involved in chemical reactions is neglected, since it is usually one or two orders of magnitude smaller than net radiation. Heat storage $G$ is considered for the soil only (the heat capacity of leaves is neglected).

The turbulent fluxes of an element $i$ are calculated from the vertical gradients of temperature and humidity for soil (index $k=1$ in the next equations) or foliage $(k=2)$ in analogy to Ohm's law for electrical current:

$H=\rho_{a} c_{p} \frac{T_{s}-T_{a}}{r_{a k}}$,

$\lambda E=\lambda \frac{q_{s}\left(T_{s}\right)-q_{a}}{r_{a k}+r_{c k}}$,

where $\rho_{a}$ is the air density $\left(\mathrm{kg} \mathrm{m}^{-3}\right), c_{p}$ the heat capacity $\left(\mathrm{J} \mathrm{kg}^{-1} \mathrm{~K}^{-1}\right), \lambda$ the evaporation heat of water $\left(\mathrm{J} \mathrm{kg}^{-1}\right), T_{s}$ the temperature of an element $\left({ }^{\circ} \mathrm{C}\right), T_{a}$ the air temperature above the canopy $\left({ }^{\circ} \mathrm{C}\right), q_{s}$ the humidity in stomata or soil pores $\left(\mathrm{kg} \mathrm{m}^{-3}\right)$ and $q_{a}$ the humidity above the canopy $\left(\mathrm{kg} \mathrm{m}^{-3}\right), r_{a}$ aerodynamic resistance and $r_{c}$ stomatal or soil surface resistance $\left(\mathrm{s} \mathrm{m}^{-1}\right)$. Both $H$ and $\lambda E$ are calculated for each surface element separately. Equation (22) holds for leaves of which only one side is contributing to transpiration (abaxial or hypostomateous leaves). In case both sides (top and bottom) of the leaf contribute to transpiration, then $r_{c k}$ in Eq. (22) is half of the one-sided value for $r_{c k}$ (Nikolov et al., 1995; Guilioni et al., 2008). 
Aerodynamic resistance $r_{a}$ is calculated with the twosource model of Wallace and Verhoef (2000). The model only differentiates between soil and foliage, and does not use separate values for aerodynamic resistance for individual leaf elements. The scalars $T$ and $q$ are calculated at the soil and leaf surfaces, at the in-canopy mixing point and the top of the roughness sublayer only. The aerodynamic resistances between these levels are calculated from the integrated reciprocal of the eddy diffusivity between the levels. The aerodynamic model is further explained in Appendix B.

Soil heat flux at the surface $G$ is calculated with the force restore method (Bhumrakhar, 1975):

$\frac{\partial T_{s}(t)}{\partial t}=\frac{\sqrt{2 \omega}}{\Gamma} G(t)-\omega\left[T_{s}(t)-\overline{T_{s}}\right]$,

where $\omega$ is the frequency of the diurnal cycle (radians $\mathrm{s}^{-1}$ ), $\Gamma$ the thermal inertia of the soil $\left(\mathrm{J} \mathrm{K}^{-1} \mathrm{~m}^{-2} \mathrm{~s}^{-1 / 2}\right)$, and $\overline{T_{S}}$ average annual temperature. The force-restore equation is discretised to:

$T_{s}(t+\Delta t)-T_{s}(t)=\frac{\sqrt{2 \omega}}{\Gamma} \Delta t G(t)-\omega \Delta t\left[T_{S}(t)-\overline{T_{S}}\right]$.

This equation is used to calculate soil temperature from the temperature at the previous time step. The fact that heat capacity of the soil is not negligible makes it necessary to simulate a time series of the fluxes in order to obtain $G$.

The energy balance is closed by adjusting skin temperatures of leaf and soil elements in an iterative manner. It is initially assumed that the skin temperatures of the elements equal the air temperature. After each iteration step, the terms $H, \lambda E, G$ and a new estimate of $T_{s}$ are calculated for each element, using the four energy balance equations (Eqs. 20, 21, 22 and 24). The aerodynamic and stomatal resistances are included in the iteration, since atmospheric stability and biochemical processes are affected by leaf temperatures. For numerical stability, a weighted average of the estimates for $T_{S}$ of the two previous iteration steps is used in the next iteration step. Iteration continues until the absolute difference in net radiation between two consecutive iterations is less than the required accuracy for all surface elements.

\subsection{Leaf biochemistry}

Leaf biochemistry affects reflectance, transmittance, transpiration, photosynthesis, stomatal resistance and chlorophyll fluorescence. Reflectance and transmittance coefficients, which are a function of the chemical composition of the leaf, are calculated with the model PROSPECT (Jacquemoud and Baret, 1990). The other variables not only depend on the chemical composition of the leaf, but also on environmental constraints such as illumination, leaf temperature and air humidity. Their nonlinear responses to environmental constraints are calculated with the model of Van der Tol et al. (2009). This model simultaneously calculates photosynthesis of C3 (Farquhar et al., 1980) or C4 vegetation (Collatz et al., 1992), stomatal resistance (Cowan, 1977) and chlorophyll fluorescence. The fluorescence module is based on conceptual understanding of the relationship between photosystem response and carboxylation. The output is the spectrally integrated level of fluorescence.

In principle, the fluorescence level $\left(\mathrm{W} \mathrm{m}^{-2}\right)$ only needs to be distributed over the spectrum $\left(\mathrm{W} \mathrm{m}^{-2} \mu \mathrm{m}^{-1}\right)$ in order to obtain the required input for the radiative transfer model: spectrally distributed leaf level fluorescence. However, the matter is complicated by two issues. First, the conceptual model is defined at organelle level. At leaf level, reabsorption of fluorescence takes place, which may reduce the fluorescence signal by an order of magnitude (Miller et al., 2005). The re-absorption varies with wavelength and with the thickness and chemical composition of the leaf. Second, the model relies on an a priori value of chlorophyll fluorescence (as a fraction of absorbed PAR) in low light conditions. This a priori value can be obtained from the literature (Genty et al., 1989), but it is unknown whether the value is universal.

To overcome these limitations, the fluorescence level is expressed as a fraction $\varphi_{f}^{\prime}$ of that of a leaf in unstressed, low light conditions. This fraction is later (in the radiative transfer model) used to linearly scale an empirically obtained matrix which converts an excitation spectrum into a fluorescence spectrum. The matrix was measured for unstressed, low light conditions. In the current version of SCOPE, two matrices are required as input: one for the upper and one for the lower side of a leaf (Sect. 2.8). In future versions of the model, the matrices might be calculated by a model similar to PROSPECT. By using the biochemical model only to describe the response to environment and not the absolute level of chlorophyll fluorescence or its spectral distribution, the problems of re-absorption and parameter estimation are circumvented.

Currently, the parameters of the biochemical model may be chosen independently from PROSPECT parameters. The parameters space could be restricted by relating PROSPECT parameters for the optical domain, such as chlorophyll content, to biochemical parameters such as photosynthetic capacity. This would make it possible to extract information about photosynthetic capacity from the optical domain.

The four most important parameters of the biochemical model are the carboxylation capacity $V_{c, \max }$, electron transport capacity $J_{\max }$, the dark respiration rate $R_{d}$ (all in $\mu \mathrm{mol} \mathrm{m}{ }^{-2} \mathrm{~s}^{-1}$ ), and the marginal water cost of photosynthesis $\lambda_{c}$. The first three parameters are temperature dependent (accounted for with Arrhenius functions; see Farquhar et al., 1980 for the exact formulation). Various studies have shown that the three parameters are correlated, and usually a constant ratio of $V_{c, \max } / J_{\max }=0.4$ is used (Wullschleger, 1993). The parameter $V_{c, \max }$ varies with depth in the canopy (Kull and Kruyt, 1998), with day of the year (Mäkelä et al., 2004) and with plant species (Wullschleger, 1993). Dark respiration and carboxylation capacity both correlate with leaf nitrogen content, but at a global scale, the correlation coefficients 
are low (Reich et al., 1999). The marginal cost of photosynthesis is a parameter to describe the compromise between the loss of water by transpiration and uptake of carbon dioxide through stomatal cavities. Parameter $\lambda_{c}$ depends on plant species and soil water potential. Weak global correlations between ecosystem type, soil water potential and $\lambda_{c}$ have been found (Lloyd and Farquhar, 1994).

\subsection{Top-of-canopy radiance spectra}

From leaf temperature, fluorescence, and the direct and diffuse fluxes at all levels in the canopy, one can calculate the top-of-canopy spectral radiances all over the spectrum. These are obtained from the spectral radiance of single leaves, by integrating the latter over canopy depth, and leaf orientation. One can also express this directly into incident fluxes, and use scattering and extinction coefficients defined for single leaves.

\subsubsection{Contributions from scattering and thermal emission}

For individual leaves, the SAIL scattering and extinction coefficients in the direction of viewing can be summarised as follows:

$$
\begin{aligned}
& \cos \delta_{s}=\cos \theta_{\ell} \cos \theta_{s}+\sin \theta_{\ell} \sin \theta_{s} \cos \varphi_{\ell} \\
& \cos \delta_{o}=\cos \theta_{\ell} \cos \theta_{o}+\sin \theta_{\ell} \sin \theta_{o} \cos \varphi \\
& \psi=\text { relative azimuth sun-view } \\
& f_{s}=\frac{\cos \delta_{s}}{\cos \theta_{s}} ; f_{o}=\frac{\cos \delta_{o}}{\cos \theta_{o}} . \\
& K=\left|f_{o}\right| \\
& v=\left|f_{o}\right| \frac{\rho+\tau}{2} \quad+f_{o} \frac{\rho-\tau}{2} \cos \theta_{\ell} \\
& v^{\prime}=\left|f_{o}\right| \frac{\rho+\tau}{2} \quad-f_{o} \frac{\rho-\tau}{2} \cos \theta_{\ell} \\
& w=\left|f_{s} f_{o}\right| \frac{\rho+\tau}{2}+f_{s} f_{o} \frac{\rho-\tau}{2}
\end{aligned}
$$$$
\cos \delta_{o}=\cos \theta_{\ell} \cos \theta_{o}+\sin \theta_{\ell} \sin \theta_{o} \cos \left(\varphi_{\ell}-\psi\right)
$$

where $K$ is the extinction coefficient in the observation direction, $v$ and $v^{\prime}$ are the scattering coefficients in the observation direction due to the contributions from downward and upward diffuse flux, respectively, and $w$ is the bi-directional scattering coefficient for solar incident radiation. The subscript $o$ in the above equations refers to the observation direction, and the subscript $s$ to the solar direction.

The TOC radiance contribution from a leaf (times $\pi$ in the direction of viewing is:

$$
\begin{aligned}
& \pi L_{\ell}=w E_{s}(0) P_{s o}(x)+ \\
& {\left[v E^{-}(x)+v^{\prime} E^{+}(x)+K \varepsilon_{c} H_{c}\left(x, \theta_{\ell}, \varphi_{\ell}\right)\right] P_{o}(x),}
\end{aligned}
$$

where $P_{o}(x)$ is the gap fraction in the observation direction (the probability to view a leaf or soil element at level $x$ from outside the canopy), and $P_{s o}(x)$ is the bi-directional gap fraction (the probability of viewing sunlit leaf or soil elements at level $x$ ). The above equation should be averaged (weighted) over all leaf orientations and split into leaf fractions in the sun and in the shade, so one obtains Eqs. (29) and (30) for the contributions from leaves in the shade and in the sun, respectively:

$$
\begin{aligned}
& \pi L_{\ell d}=\sum_{13 \theta_{\ell}}\left\{\left[v E^{-}(x)+v^{\prime} E^{+}(x)\right] P_{o}(x)\left[1-P_{S}(x)\right]+\right. \\
& 36 \varphi_{\ell} \\
& 60 x \\
& \left.K \varepsilon_{c} H_{c d}(x)\left[P_{o}(x)-P_{s o}(x)\right]\right\} f\left(\theta_{\ell}\right) / 36 \times \frac{L}{60} \\
& \pi L_{\ell s}=\sum_{\substack{13 \theta_{\ell} \\
36 \varphi_{\ell}}}\left\{\begin{array}{l}
{\left[w E_{s}(0)+K\left(\theta_{\ell}, \varphi_{\ell}\right) \varepsilon_{c} H_{c s}\left(x, \theta_{\ell}, \varphi_{\ell}\right)\right] P_{s o}(x)} \\
+\left[v E^{-}(x)+v^{\prime} E^{+}(x)\right] P_{o}(x) P_{s}(x)
\end{array}\right\} \\
& 60 x \\
& f\left(\theta_{\ell}\right) / 36 \times \frac{L}{60} .
\end{aligned}
$$

This assumes there are 36 leaf azimuth angles and 60 layers. The above equations can be decomposed in quantities that depend either on the leaf orientation or the level. The weighted averaging over the leaf inclination and azimuth could be done first, and next the mean values (these are the analytical SAIL coefficients) could be used in the summation over levels. However, $K$ in Eq. (30) must still be differentiated according to leaf orientation, since the leaves' thermal emittances vary with leaf orientation as well.

The bidirectional gap fraction, which is the probability of observing a sunlit leaf at depth $x$ in the canopy is given by

$$
P_{s o}(x)=\exp \left[(K+k) x+\sqrt{K k} \frac{\ell}{\beta}\left(1-e^{x \frac{\beta}{\ell}}\right)\right],
$$

where $\ell$ is the ratio of leaf width to canopy height, and

$\beta=\sqrt{\tan ^{2} \theta_{s}+\tan ^{2} \theta_{o}-2 \tan \theta_{s} \tan \theta_{o} \cos \psi}$.

Finally, the contributions from the soil background (sunlit and shaded) should be added. They are given by

$$
\begin{aligned}
& \pi L_{s s}=\left\{r_{s}\left[E^{-}(-1)+E_{s}(0)\right]+\varepsilon_{s} H_{s s}\right\} P_{s o}(-1) \\
& \pi L_{s d}=\left[r_{s} E^{-}(-1)+\varepsilon_{s} H_{s d}\right]\left[P_{o}(-1)-P_{s o}(-1)\right] .
\end{aligned} .
$$

The sum is given by

$$
\begin{aligned}
\pi L_{s}= & {\left[r_{s} E^{-}(-1)+\varepsilon_{s} H_{s d}\right] P_{o}(-1) } \\
& +\left[r_{s} E_{S}(0)+\varepsilon_{s}\left(H_{s s}-H_{s d}\right)\right] P_{s o}(-1) .
\end{aligned}
$$

In Eq. (34), $P_{o}(x)$ is the probability of observing a leaf at depth $x$. In the final result, frequent use is made of the analytical expressions for the scattering coefficients from the SAIL model, so that in the numerical calculation mostly only a summation over the 60 layers needs to be done, as can be seen from Eq. (35). There is only one term left for which a 
summation over leaf orientations as well as depth level has to be made.

$$
\begin{aligned}
& \pi L_{o}(0)= \\
& {\left[\begin{array}{l}
v \sum_{60 x} E^{-}(x) P_{o}(x)+v^{\prime} \sum_{60 x} E^{+}(x) P_{o}(x)+ \\
K \varepsilon_{c} \sum_{60 x} H_{c d}(x)\left[P_{o}(x)-P_{s o}(x)\right] \\
+w E_{S}(0) \sum_{60 x} P_{s o}(x)+ \\
\varepsilon_{c} \sum_{1} K\left(\theta_{\ell}, \varphi_{\ell}\right) H_{c s}\left(x, \theta_{\ell}, \varphi_{\ell}\right) f\left(\theta_{\ell}\right) P_{s o}(x) / 36 \\
13 \theta_{\ell} \\
36 \varphi_{\ell} \\
60 x
\end{array}\right] \frac{L}{60}} \\
& +\left[r_{s} E^{-}(-1)+\varepsilon_{s} H_{s d}\right] P_{o}(-1)+ \\
& {\left[r_{s} E_{s}(0)+\varepsilon_{s}\left(H_{s s}-H_{s d}\right)\right] P_{s o}(-1)}
\end{aligned}
$$

Equation (35) can be calculated more efficiently when the analytical SAIL model is used for the contributions from solar and sky irradiance, excluding the internally generated thermal radiation. The terms in Eq. (35) containing the SAIL coefficients $v, v^{\prime}, w$ and $r_{s}$ together form the directional reflectance contribution from the canopy and soil. Using the canopy-level reflectances for direct and diffuse radiation in observation direction $r_{s o}$ and $r_{d o}$, Eq. (35) can be re-written as:

$$
\begin{aligned}
& \pi L_{o}(0)=r_{s o} E_{\mathrm{sun}}+r_{d o} E_{\mathrm{sky}}+ \\
& {\left[\begin{array}{c}
+K \varepsilon_{c} \sum_{60 x} H_{c d}(x)\left[P_{o}(x)-P_{s o}(x)\right] \\
+\varepsilon_{c} \sum_{13 \theta_{\ell}}\left(\theta_{\ell}, \varphi_{\ell}\right) H_{c s}\left(x, \theta_{\ell}, \varphi_{\ell}\right) f\left(\theta_{\ell}\right) P_{s o}(x) / 36 \\
36 \varphi_{\ell} \\
60 x \\
+\varepsilon_{s} H_{s d} P_{o}(-1)+\varepsilon_{s}\left(H_{s s}-H_{s d}\right) P_{s o}(-1)
\end{array}\right] \frac{L}{60}}
\end{aligned}
$$

\subsubsection{Contribution from leaf fluorescence}

Fluorescence from single leaves is calculated with the biochemical module (Sect. 2.7) using the absorbed fluxes over the PAR region $(400-700 \mathrm{~nm})$. In addition, two excitationfluorescence matrices (EF-matrices) must be given to represent fluorescence from both sides of the leaf, which have been experimentally derived for unstressed, low light conditions. The matrices convert a spectrum of absorbed PAR into a spectrum of fluorescence. The fluorescence matrices are linearly scaled for each element with a factor $\phi_{f}^{\prime}$ and with incident PAR to obtain the actual fluorescence.

Absorbed PAR of direct ( $\left.E_{\text {ap,dir }}\right)$ and diffuse light ( $\left.E_{\text {ap,dif }}\right)$ can be calculated by integrating incident radiation over the PAR wavelength range, $\lambda$, as follows:

$$
E_{\text {ap }, \text { dir }}=\int_{400}^{700} E_{\text {sun }}(\lambda)[1-\rho(\lambda)-\tau(\lambda)] \mathrm{d} \lambda
$$

$$
\begin{gathered}
E_{\text {ap }, \operatorname{dif}}(x)=\int_{400}^{700}\left[E^{-}(x, \lambda)+E^{+}(x, \lambda)\right] \\
\\
{[1-\rho(\lambda)-\tau(\lambda)] \mathrm{d} \lambda .}
\end{gathered}
$$

The second expression can be used directly to obtain the absorbed PAR radiation by leaves in the shade $\left(E_{a p, d}\right)$. For leaves in the sun, also their orientation must be taken into account, and one so obtains

$E_{a p, s}\left(x, \theta_{\ell}, \varphi_{\ell}\right)=\left|f_{s}\right| E_{\mathrm{ap}, \mathrm{dir}}+E_{\mathrm{ap}, \mathrm{dif}}(x)$.

Application of the photosynthesis-fluorescence model of Van der Tol et al. (2009) yields fluorescence amplification factors $\phi_{f s}^{\prime}\left(x, \theta_{\ell}, \varphi_{\ell}\right)$ and $\phi_{f d}^{\prime}(x)$ for leaves in the sun and in the shade, respectively, that should be treated as correction factors applied to the EF-matrices, which determine the spectral distribution of the fluorescent flux. The EF-matrices are symbolised as $M\left(\lambda_{e}, \lambda_{f}\right)$ and $M^{\prime}\left(\lambda_{e}, \lambda_{f}\right)$ for backward and forward fluorescence, respectively.

For the fluorescent radiance response to incident light for leaves in the sun with a particular orientation one can write

$$
\begin{aligned}
& \pi L_{\ell s}^{f}\left(x, \lambda_{f}, \theta_{\ell}, \varphi_{\ell}\right)=\phi_{f s}^{\prime}\left(x, \theta_{\ell}, \varphi_{\ell}\right) \int_{400}^{750} \\
& {\left[\begin{array}{c}
w_{f}\left(\lambda_{e}, \lambda_{f}, \theta_{\ell}, \varphi_{\ell}\right) E_{\text {sun }}\left(\lambda_{e}\right) \\
+v_{f}\left(\lambda_{e}, \lambda_{f}, \theta_{\ell}, \varphi_{\ell}\right) E^{-}\left(x, \lambda_{e}\right) \\
+v_{f}^{\prime}\left(\lambda_{e}, \lambda_{f}, \theta_{\ell}, \varphi_{\ell}\right) E^{+}\left(x, \lambda_{e}\right)
\end{array}\right] \mathrm{d} \lambda_{e} .}
\end{aligned}
$$

Here it was assumed that the range of excitation wavelengths is from 400 to $750 \mathrm{~nm}$. The coefficients are defined by analogy with Eqs. (27) and are given by

$$
\begin{aligned}
& v_{f}\left(\lambda_{e}, \lambda_{f}, \theta_{\ell}, \varphi_{\ell}\right)= \\
& \left|f_{o}\right| \frac{M\left(\lambda_{e}, \lambda_{f}\right)+M^{\prime}\left(\lambda_{e}, \lambda_{f}\right)}{2}+f_{o} \frac{M\left(\lambda_{e}, \lambda_{f}\right)-M^{\prime}\left(\lambda_{e}, \lambda_{f}\right)}{2} \cos \theta_{\ell} \\
& v_{f}^{\prime}\left(\lambda_{e}, \lambda_{f}, \theta_{\ell}, \varphi_{\ell}\right)= \\
& \left|f_{o}\right| \frac{M\left(\lambda_{e}, \lambda_{f}\right)+M^{\prime}\left(\lambda_{e}, \lambda_{f}\right)}{2}-f_{o} \frac{M\left(\lambda_{e}, \lambda_{f}\right)-M^{\prime}\left(\lambda_{e}, \lambda_{f}\right)}{2} \cos \theta_{\ell} \\
& w_{f}\left(\lambda_{e}, \lambda_{f}, \theta_{\ell}, \varphi_{\ell}\right)= \\
& \left|f_{s} f_{o}\right| \frac{M\left(\lambda_{e}, \lambda_{f}\right)+M^{\prime}\left(\lambda_{e}, \lambda_{f}\right)}{2}+f_{s} f_{o} \frac{M\left(\lambda_{e}, \lambda_{f}\right)-M^{\prime}\left(\lambda_{e}, \lambda_{f}\right)}{2}
\end{aligned}
$$

For leaves in the shade the fluorescent radiance can be described by

$$
\begin{aligned}
& \pi L_{\ell d}^{f}\left(x, \lambda_{f}\right)=\phi_{f d}^{\prime}(x) \int_{400}^{750} \\
& {\left[v_{f}\left(\lambda_{e}, \lambda_{f}\right) E^{-}\left(x, \lambda_{e}\right)+v_{f}^{\prime}\left(\lambda_{e}, \lambda_{f}\right) E^{+}\left(x, \lambda_{e}\right)\right] \mathrm{d} \lambda_{e},}
\end{aligned} .
$$


where both fluorescent scattering coefficients are supposed to have been obtained by weighted averaging over all leaf orientations, i.e.

$$
\begin{aligned}
v_{f}\left(\lambda_{e}, \lambda_{f}\right) & =\frac{1}{36} \sum_{13 \theta_{\ell}} f\left(\theta_{\ell}\right) \sum_{36 \varphi_{\ell}} v_{f}\left(\lambda_{e}, \lambda_{f}, \theta_{\ell}, \varphi_{\ell}\right) \\
v_{f}^{\prime}\left(\lambda_{e}, \lambda_{f}\right) & =\frac{1}{36} \sum_{13 \theta_{\ell}} f\left(\theta_{\ell}\right) \sum_{36 \varphi_{\ell}} v_{f}^{\prime}\left(\lambda_{e}, \lambda_{f}, \theta_{\ell}, \varphi_{\ell}\right) .
\end{aligned}
$$

The total top-of-canopy fluorescent radiance is now obtained by a summation over all layers and orientations, taking into account the probabilities of viewing sunlit and shaded components. This gives

$$
\begin{aligned}
& \pi L_{f}^{\mathrm{TOC}}= \\
& \frac{L}{60} \sum_{60 x}\left[\frac{P_{s o}(x)}{36} \sum_{13 \theta_{\ell}} f\left(\theta_{\ell}\right) \sum_{36 \varphi_{\ell}} \pi L_{\ell s}^{f}\left(x, \lambda_{f}, \theta_{\ell}, \varphi_{\ell}\right)+\right. \\
& \left.\left[P_{o}(x)-P_{s o}(x)\right] \pi L_{\ell d}^{f}\left(x, \lambda_{f}\right)\right] .
\end{aligned}
$$

\section{Output of SCOPE}

\subsection{Spectra}

It is not necessary to run SCOPE with the same spectral resolution as the input data, but the resolution of the input data obviously affects the accuracy of the output of SCOPE. Input data with a high spectral resolution are not always available. In the absence of spectral input data, spectra could be selected from a library of MODTRAN4 runs for specific weather conditions, and scaled in such a way that the integrated radiation agrees with broadband measurements. As an example, Fig. 2 shows an input spectrum for SCOPE, calculated with MODTRAN4, run at a spectral resolution of $1 \mathrm{~cm}^{-1}$ in wavenumbers, which gives a spectral resolution ranging from $0.02 \mathrm{~nm}$ in the optical to $250 \mathrm{~nm}$ in the thermal domain. This example has been used as input spectrum for the simulations presented in this paper.

Figure 3 shows examples of model output: output radiance spectra in nadir direction, calculated using input data collected during a field experiment in Sonning, United Kingdom (Houldcroft, 2004). Two scenarios are shown: a typical fully grown maize crop (LAI $=3.22)$ and a sparse maize crop $(\mathrm{LAI}=0.25)$. As meteorological input, a wind speed of $2.9 \mathrm{~m} \mathrm{~s}^{-1}$ was used, an absolute humidity of $q_{a}=9.3 \mathrm{~g} \mathrm{~kg}^{-1}$ and air temperature $T_{a}=21.4^{\circ} \mathrm{C}$. Measured data were used for the sake of providing realistic input values, but no validation data for the spectra were available as these were not measured. Other model parameters are listed in Table 2. The input spectra of Fig. 2 were used, albeit linearly scaled such that total incoming shortwave $(0.4-2.5 \mu \mathrm{m})$ radiation matched the measured value of $646 \mathrm{~W} \mathrm{~m}^{-2}$. The upper graph shows the results for the optical range (excluding fluorescence), the middle graph for the thermal range, and the lower graph shows chlorophyll fluorescence.
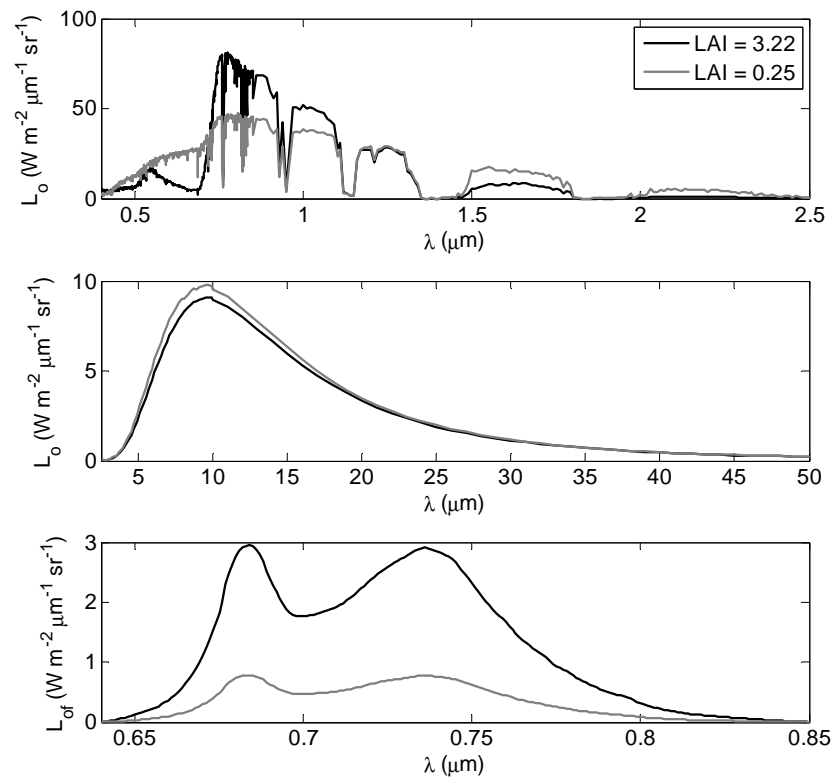

Fig. 3. Outgoing TOC optical to NIR (upper graph), thermal (middle graph) and chlorophyll fluorescence radiance (lower graph) in nadir direction, for two scenarios (low LAI and high LAI) of a C4 canopy. The relevant parameters are listed in Table 2.

Table 2. The most relevant parameters used for the SCOPE simulations for a $\mathrm{C} 4$ canopy. Parameters $C_{a b}, C_{d m}, C_{s}$ and $N$ are PROSPECT parameters (Jacquemoud, 1990), and refer to chlorophyll content, dry matter content, senescent material, and leaf structure, respectively. $\mathrm{LIDF}_{a}$ and $\mathrm{LIDF}_{b}$ are leaf angle distribution parameters (Verhoef et al., 2007). The values in the table refer to a spherical distribution of leaves. Other parameters are explained in Table 1.

\begin{tabular}{lcl}
\hline Parameter & Value & Unit \\
\hline$C_{a b}$ & 60 & $\mu \mathrm{g} \mathrm{cm}^{-2}$ \\
$C_{d m}$ & 0.012 & $\mathrm{~g} \mathrm{~cm}^{-2}$ \\
$C_{w}$ & 0.009 & $\mathrm{~cm}$ \\
$C_{s}$ & 0.0 & \\
$N$ & 1.4 & \\
$\mathrm{LIDF}_{a}$ & -0.35 & \\
$\mathrm{LIDF}_{b}$ & -0.15 & \\
$\mathrm{LAI}$ & $3.22 / 0.25$ & \\
$V_{\mathrm{cmax}}$ & 50 & $\mu \mathrm{mol} \mathrm{m}^{-2} \mathrm{~s}^{-1}$ \\
$V_{\mathrm{pmo}}$ & 220 & $\mu \mathrm{mol} \mathrm{m}^{-2} \mathrm{~s}^{-1}$ \\
$J_{\text {max }}$ & 120 & $\mu \mathrm{mol} \mathrm{m}^{-2} \mathrm{~s}^{-1}$ \\
$\lambda_{c}$ & 700 & \\
$h$ & 1.0 & $\mathrm{~m}$ \\
$\rho$ (thermal) & 0.01 & \\
$\tau$ (thermal) & 0.01 & \\
$\rho_{S}$ (thermal) & 0.06 & \\
\hline
\end{tabular}



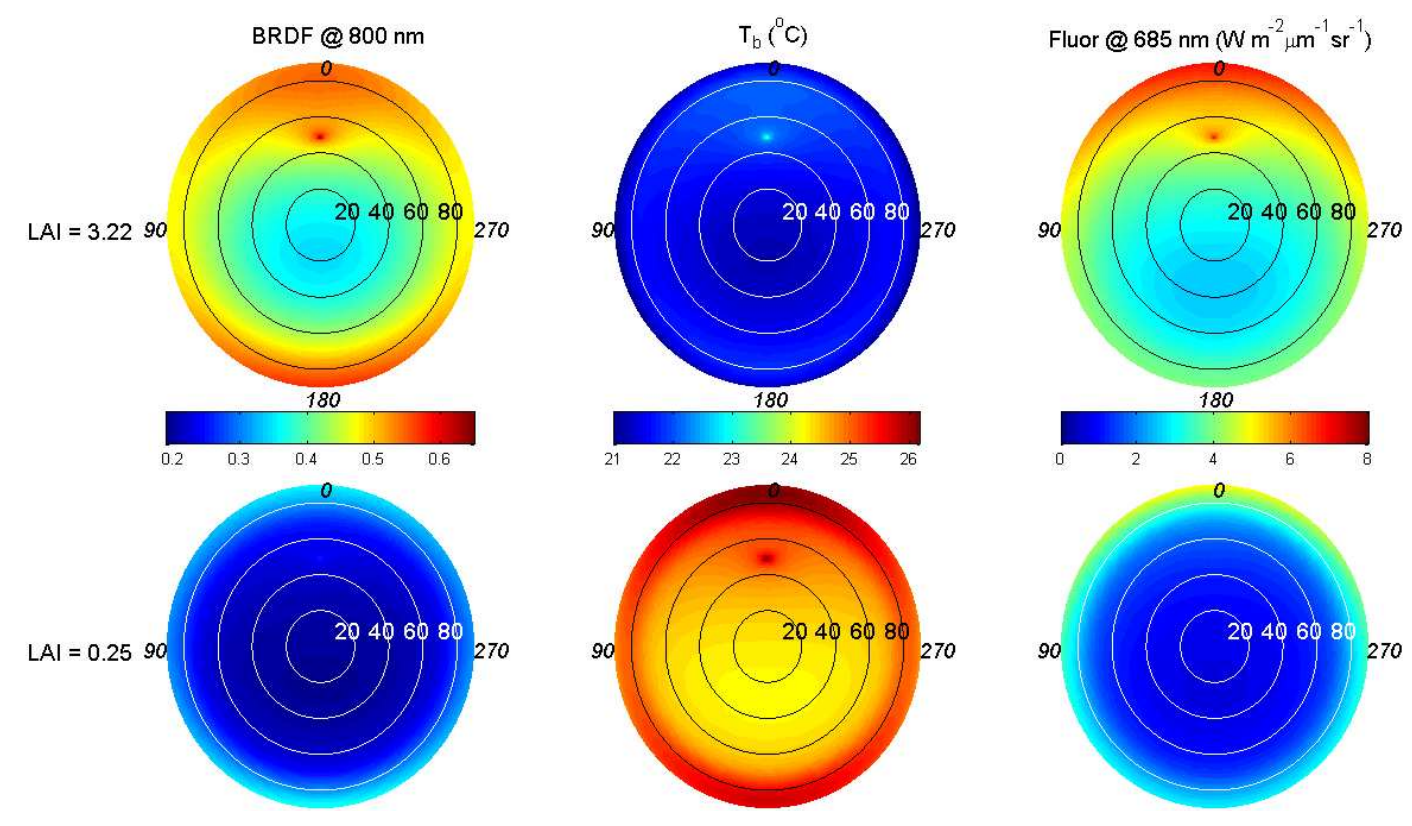

Fig. 4. For the same scenarios as in Fig. 3, hemispherical graphs of top-of-canopy reflectance (left), brightness temperature (middle) and chlorophyll fluorescence radiance (right) as a function of viewing zenith angle and viewing azimuth angle (relative to the solar azimuth). Zenith angle varies with the radius, the azimuth angle (in italic) increases while rotating anticlockwise from north. The solar zenith angle was $48^{\circ}$.

The optical spectrum of the fully grown canopy is typical for green vegetation (Carter and Knapp, 2001), whereas the spectrum for the sparse canopy represents a mixture of bare soil and vegetation.

While the optical spectrum is only a function of irradiance and leaf and canopy characteristics, the thermal spectrum also depends on canopy and soil temperatures, and thus on the energy balance. Thermal radiation includes radiation emitted by the soil and the vegetation as well as a small contribution of reflected sky radiation. In the presented scenarios, the thermal spectrum closely resembles Planck's curve, because emissivity was not differentiated spectrally. The differences between the two scenarios are the combined result of differences in emissivity, reflected thermal radiation, and canopy and soil temperatures. In the sparse canopy, a larger portion of the soil is visible. The soil has a lower emissivity than the leaf, thus reducing the radiance of the sparse canopy compared to the fully grown canopy. This is compensated by the fact that both soils and leaves have higher temperature in the sparse canopy than in the fully grown canopy.

The chlorophyll fluorescence spectra show two peaks, one in the red $(690 \mathrm{~nm})$ and one in the far red $(730 \mathrm{~nm})$. The ratio between the first and the second peak usually decreases with increasing chlorophyll content due to re-absorption within the leaf (Buschmann, 2007). In the current simulations, the re-absorption within the leaf is not included, as the matrices for the upside and downside of a leaf were prescribed as input. However, the (minor) effect of re-absorption of other leaf layers is included, which causes the ratio of the first over the second peak to be slightly higher in the sparse canopy compared to the fully grown canopy. The magnitude of the fluorescence fluxes agrees with recent measurements: Entcheva Campbell et al. (2008) found peak values of $2-5 \mathrm{~W} \mathrm{~m}^{-2} \mu \mathrm{m}^{-1} \mathrm{sr}^{-1}$ for a maize crop.

\subsection{Directional radiance}

SCOPE was designed to simulate directional effects, i.e. the dependence of the measurement on the observation angle. Figure 4 shows the directional behaviour of reflectance, brightness temperature and fluorescence for the two scenarios presented in Fig. 3. Directional simulations can serve as a tool to study the added value of multi-angular observations.

The hotspot is clearly visible for the BRDF at $800 \mathrm{~nm}$ and for the brightness temperature. Even for fluorescence it is visible. In the hot spot one observes only sunlit elements and this has a clear impact in all domains.

The sparse crop has higher brightness temperatures, although the brightness temperature is lower than the actual temperatures of leaves and soil (Fig. 3) due to the relatively low emissivity of the soil ( 0.94 compared to 0.98 for leaves). Brightness temperature observed with angles perpendicular to the solar azimuth angles are relatively low compared to those measured in the principal plane. While increasing the viewing angle (from vertical to horizontal) the brightness temperature increases in concert. The reason is that at horizontal viewing angles, the largest fraction of (relatively warm) sunlit vegetation is visible. This effect is most 

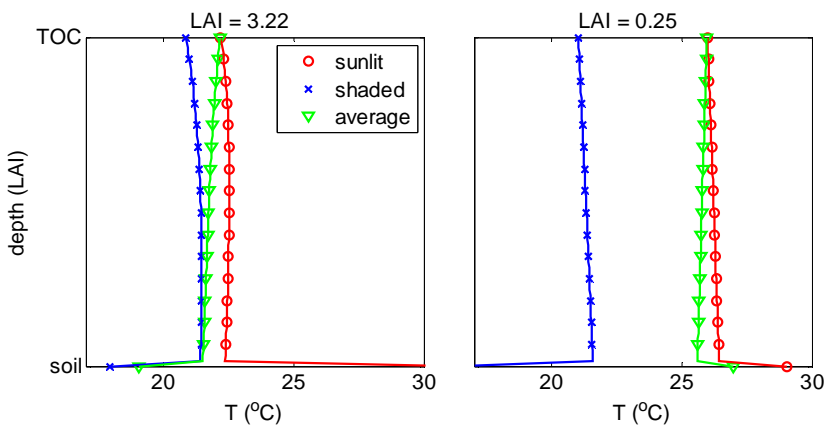

Fig. 5. For the same scenarios as in Fig. 3, vertical profiles of contact temperatures of leaves and soil (averages per layer). The top of the graph represents the top of canopy; the bottom of the graph represents the soil. Temperatures are contact (skin) temperatures of the canopy, except for the values at the bottom of the graph: these are contact temperatures of the soil. The vertical axis scales linearly with leaf area index.

pronounced in the sparse vegetation, because there the differences in temperature between sunlit and shaded vegetation are the largest (Fig. 5).

The directional effect is quite pronounced for chlorophyll fluorescence. The upper leaves contribute most to total chlorophyll fluorescence, and as a result, observed fluorescence increases when moving from vertical to horizontal viewing angles.

Note that clumping of leaves, twigs and branches may also affect the directional effects in forest canopies (Smolander and Stenberg, 2003). This effect is not included at present, but it may be a feature of future versions of the model.

\subsection{Vertical profiles}

One of the best ways to illustrate the integration of radiative transfer with the energy balance is by plotting vertical profiles of the canopy. Figure 5 shows, for the two scenarios of Fig. 3, vertical profiles of leaf and soil surface temperature in the canopy. Values represent the average per layer, for the sunlit fraction, the shaded fraction, and the weighted average temperature. The bottom layer is the soil.

The temperature of both sunlit and shaded leaves increases with depth in the canopy (from top to bottom of the graph), whereas the weighted mean temperature decreases with depth, due to the fact that the shaded fraction progressively dominates while moving to lower layers. The sparse canopy has higher leaf temperatures than the fully grown canopy, both for the shaded and the sunlit fraction. This effect is caused by the high net radiation on the soil, resulting in higher soil contact temperatures, which also affect the canopy layer above through a higher emittance received from below. The weighted average of leaf and soil temperatures is also higher for the sparse vegetation, because the fraction of sunlit leaves is higher.

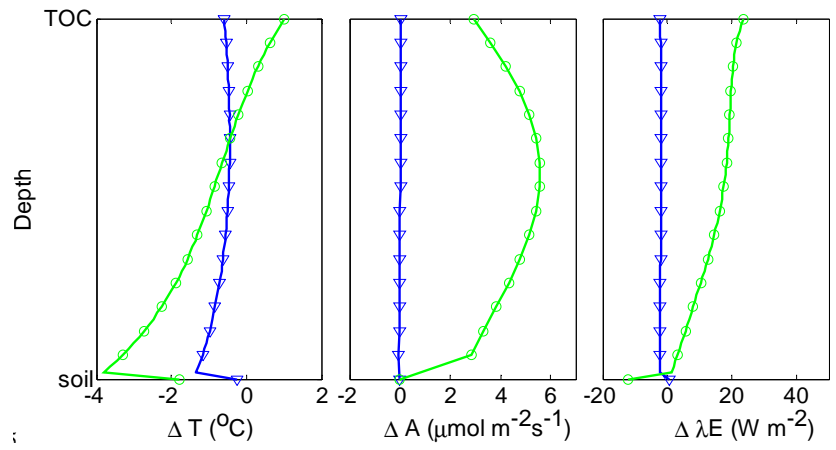

Fig. 6. The effect of increasing wind speed from $2.9 \mathrm{~m} \mathrm{~s}^{-1}$ to $15 \mathrm{~m} \mathrm{~s}^{-1}$ (blue line and triangles), and the effect of replacing all direct radiation by diffuse radiation -while total radiation remains unchanged- (green line with circles), for the fully grown maize canopy of Fig. 3. The graphs show the difference in the vertical profiles of leaf and soil temperatures, net photosynthesis and latent heat flux compared to the reference scenario.

The vertical profiles are sensitive to variations in irradiance regime as well as weather conditions. Different vertical profiles of the fully grown maize field resulted by (1) a fivefold higher wind speed $\left(15 \mathrm{~m} \mathrm{~s}^{-1}\right)$, and (2) replacing all direct radiation by diffuse radiation (Fig. 6). Not only the vertical profiles of temperature change, but also those of photosynthesis and latent heat.

Wind speed has a significant effect on temperature, but only minor effect on the fluxes: the decrease in aerodynamic resistances as wind speed increases is counterbalanced by a simultaneous decrease in the vapour and carbon dioxide gradients. The distribution of radiation over direct and diffuse radiation has a significant effect on both the temperature and the fluxes. This results confirms that photosynthetic light use efficiency is higher for diffuse than for direct radiation $(\mathrm{Gu}$ et al., 2002).

\section{Applications of SCOPE}

In this section, a number of simulations are presented to address the potential and the limitations of the SCOPE model. Most of the individual components of the model have been validated before, such as the optical radiative transfer model (Jacquemoud et al., 2000) and the leaf physiological model (Von Caemmerer and Baker, 2007). A validation of all components of the model against field data will be the topic of a follow-up paper.

\subsection{Plant physiology}

SCOPE has potential applications for plant physiology and carbon uptake. This is illustrated in Fig. 7, showing measured (with an ASD radiometer) and simulated reflectance spectra of a Pine forest in The Netherlands. Data were 


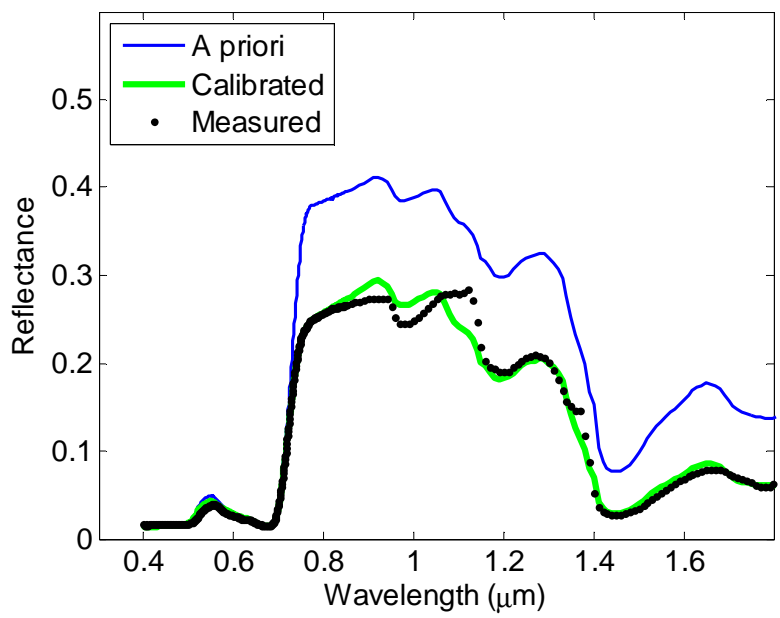

Fig. 7. Measured and simulated spectrum of radiance versus wavelength for a Pine forest in The Netherlands, 13 June 2006 at 11:45 local (winter) time $(\mathrm{GMT}+1)$. Measurements were taken with an ASD spectrometer from a $46 \mathrm{~m}$ tower raising $16 \mathrm{~m}$ above the canopy (solar zenith angle $\theta_{s}=30.6^{\circ}$, solar azimuth angle $\phi_{S}=345^{\circ}$, observer zenith angle $\theta_{O}=30^{\circ}$, observer azimuth angle $\phi_{o}=185^{\circ}$ ). A priori parameter values of Table 2 were used. Calibration was carried out using a non-linear least squares solver. Calibrated PROSPECT parameters were: $C_{a b}=47 \mu \mathrm{g} \mathrm{cm}^{-2}$, $C_{d m}=0.025 \mathrm{~g} \mathrm{~cm}^{-2}, C_{w}=0.0195 \mathrm{~cm}, C_{s}=0.114, N=1.07$.

collected during an intensive field campaign (EAGLE) in June 2006, described in detail by Su et al. (2009). Simulations were carried out both using a priori, literature values for the PROSPECT parameters (Table 2), and using calibrated PROSPECT parameters (calibration of SCOPE was carried out using a non-linear least square solver). Fitting the model to observations resulted in PROSPECT parameter values for chlorophyll content and leaf water content that were different from the a priori values. Although currently no model exists to relate PROSPECT parameters to plant physiological processes such as photosynthesis, it is theoretically possible to limit the parameter space of SCOPE by linking calibrated optical parameters of PROSPECT to parameters of the biochemical leaf model. Such approach would greatly improve the remote sensing of physiology and carbon uptake.

\subsection{Testing of surface energy balance models}

A different application is to use SCOPE to evaluate and improve simpler, operational remote sensing based surface energy balance models, such as SEBAL (Bastiaanssen, 1998) or SEBS (Su, 2002). The use of SCOPE as a hypothetical ground truth not only provides the necessary validation data, but also simulates the input data, notably the TOC radiative spectra. By using these simulated spectra, complica- tions due to differences in scale between ground and remote sensing images and issues of atmospheric correction are circumvented.

An example is given in Fig. 8. This figure shows on the left, simulated input, and on the right, simulated output for the model SEBS. The left graphs shows the difference between brightness temperature (calculated from outgoing long wave radiance), and air temperature measured above the canopy. The input data for this simulation were collected during a field experiment in Barrax (Spain) on 17 July 2004 (Su et al., 2008). Field measurements for validation are also shown in the graph. Brightness temperature is the most important input variable of SEBS, but other variables such as NDVI can be simulated with SCOPE as well.

\subsection{Interpolation between satellite overpasses}

A related possible application of SCOPE is to interpolate fluxes between satellite overpasses. The model can run in the absence of remote sensing information, which makes it possible to not only scale from instantaneous data to diurnal cycles, but also to calculate the fluxes for clouded days, for which reliable remote sensing data are not available.

\subsection{The use of chlorophyll fluorescence}

The use of chlorophyll fluorescence signal is another promising application. The SCOPE model contains parameters which control the amount of fluorescence per leaf as a function of leaf physiological parameters like $V_{c, \max }$ and $\lambda$, the optical parameters of the PROSPECT model, such as the chlorophyll content, and light and temperature conditions inside the canopy. Since leaf photosynthesis is included in the modelling as well, one could investigate the relationships between light use efficiency and fluorescence at the canopy level under different simulated conditions (stress, canopy structure, weather, etc.). This could be of great help for the correct interpretation of fluorescence measurements from a satellite mission like FLEX (Rascher et al., 2008).

\section{Conclusions}

SCOPE integrates radiative transfer and energy balance calculations at the level of individual leaves as well as the canopy. Potential applications are in plant physiology, remote sensing of the energy balance, and the preparation of future satellite missions such as the fluorescence explorer mission FLEX. The modular structure of SCOPE makes it possible to add new features by simply sharing input, output and parameters with other models. Future developments include the adding a library of MODTRAN output spectra for various weather conditions, as well as a library of soil spectra. 

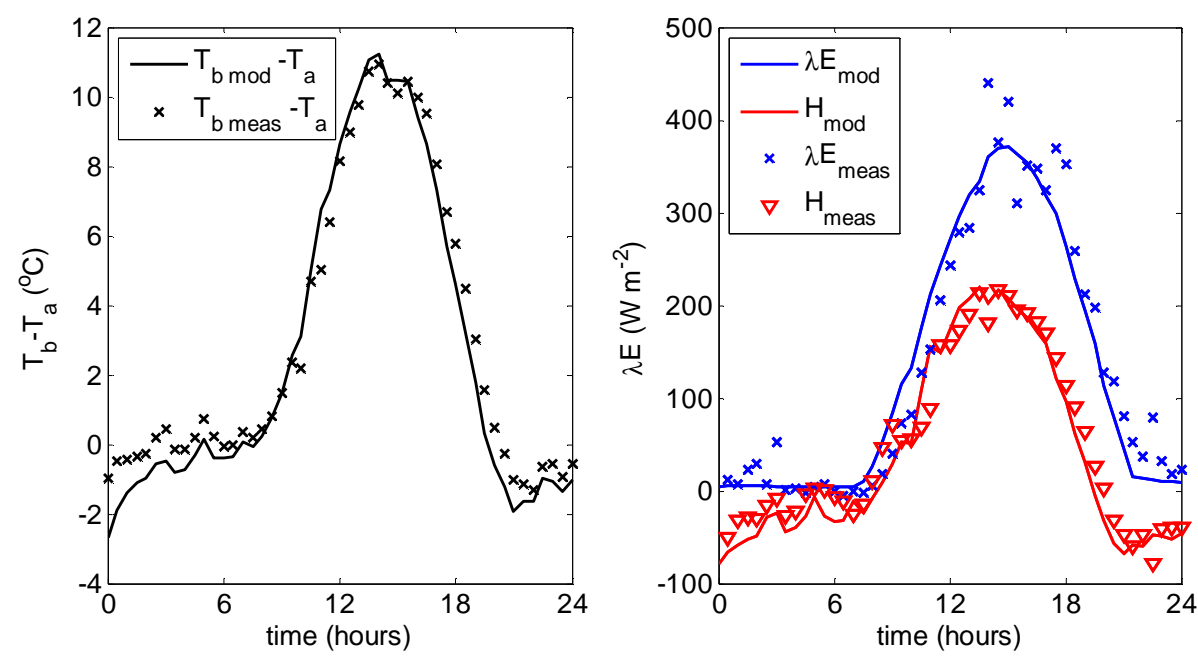

Fig. 8. Measured and simulated diurnal cycles of brightness temperature, latent and sensible heat flux for a Vineyard in Barrax, Spain, 17 July 2004. Measured brightness temperature was based on measurements of a radiometer above the canopy, latent and sensible heat flux on measurements with a sonic anemometer and open path gas analyser.

\section{Appendix A}

\section{Numerically stable fluxes in the 4SAIL model}

The two-stream radiative transfer equation in matrix-vector form reads

$\frac{\mathrm{d}}{L \mathrm{~d} x}\left(\begin{array}{c}E^{-} \\ E^{+}\end{array}\right)=\left(\begin{array}{c}a-\sigma \\ \sigma-a\end{array}\right)\left(\begin{array}{l}E^{-} \\ E^{+}\end{array}\right)$.

With the eigenvalue $m=\sqrt{(a-\sigma)(a+\sigma)}$, one can define the so-called infinite reflectance, which is given by $r_{\infty}=\frac{a-m}{\sigma}$, and by means of the left-hand eigenvector matrix $\left(\begin{array}{cc}1 & -r_{\infty} \\ -r_{\infty} & 1\end{array}\right)$ one can define transformed fluxes given by

$$
\left(\begin{array}{l}
F_{1} \\
F_{2}
\end{array}\right)=\left(\begin{array}{cc}
1 & -r_{\infty} \\
-r_{\infty} & 1
\end{array}\right)\left(\begin{array}{l}
E^{-} \\
E^{+}
\end{array}\right) .
$$

This transformation establishes the diagonalization of the two-stream radiative transfer matrix, since

$$
\begin{aligned}
\frac{\mathrm{d}}{L \mathrm{~d} x}\left(\begin{array}{l}
F_{1} \\
F_{2}
\end{array}\right) & =\left(\begin{array}{cc}
1 & -r_{\infty} \\
-r_{\infty} & 1
\end{array}\right)\left(\begin{array}{ll}
a & -\sigma \\
\sigma & -a
\end{array}\right)\left(\begin{array}{l}
E^{-} \\
E^{+}
\end{array}\right) \\
& =\left(\begin{array}{cc}
m & 0 \\
0 & -m
\end{array}\right)\left(\begin{array}{cc}
1 & -r_{\infty} \\
-r_{\infty} & 1
\end{array}\right)\left(\begin{array}{l}
E^{-} \\
E^{+}
\end{array}\right) \\
& =\left(\begin{array}{cc}
m & 0 \\
0 & -m
\end{array}\right)\left(\begin{array}{l}
F_{1} \\
F_{2}
\end{array}\right) .
\end{aligned}
$$

Addition of direct solar flux and thermal emittance by the foliage to Eq. (A1) gives

$$
\begin{aligned}
\frac{\mathrm{d}}{L \mathrm{~d} x}\left(\begin{array}{c}
E^{-} \\
E^{+}
\end{array}\right) & =E_{s}(0) e^{k L x}\left(\begin{array}{c}
-s^{\prime} \\
s
\end{array}\right)+\left(\begin{array}{c}
a-\sigma \\
\sigma-a
\end{array}\right)\left(\begin{array}{c}
E^{-} \\
E^{+}
\end{array}\right) \\
& +\varepsilon_{v}\left(\begin{array}{c}
-H_{c} \\
H_{c}
\end{array}\right) .
\end{aligned}
$$

The transformation by the left-hand eigenvector matrix can now be applied again to obtain

$$
\begin{aligned}
\frac{\mathrm{d}}{L \mathrm{~d} x}\left(\begin{array}{c}
F_{1} \\
F_{2}
\end{array}\right) & =E_{s}(0) e^{k L x}\left(\begin{array}{c}
-s^{\prime}-r_{\infty} s \\
r_{\infty} s^{\prime}+s
\end{array}\right)+\left(\begin{array}{cc}
m & 0 \\
0 & -m
\end{array}\right)\left(\begin{array}{c}
F_{1} \\
F_{2}
\end{array}\right) \\
& +\varepsilon_{v}\left(1+r_{\infty}\right)\left(\begin{array}{c}
-H_{c} \\
H_{c}
\end{array}\right) .
\end{aligned}
$$

One can write

$$
\begin{aligned}
\frac{1+r_{\infty}}{1-r_{\infty}} & =\frac{1+\frac{a-m}{\sigma}}{1-\frac{a-m}{\sigma}}=\frac{\sigma+a-m}{\sigma-a+m}=\frac{m(a+\sigma-m)}{m^{2}-m(a-\sigma)} \\
& =\frac{m(a+\sigma-m)}{(a-\sigma)(a+\sigma-m)}=\frac{m}{a-\sigma} .
\end{aligned}
$$

Since $a-\sigma=\alpha=\varepsilon_{c}$ (Kirchhoff's law), where $\alpha$ is the absorption coefficient and $\varepsilon_{c}$ the foliage emissivity, one can write $\left(1+r_{\infty}\right) \varepsilon_{c}=m\left(1-r_{\infty}\right)$, so finally the following differential equations are obtained:

$$
\begin{aligned}
\frac{\mathrm{d}}{L \mathrm{~d} x} F_{1} & =m F_{1}-\left(s^{\prime}+r_{\infty} s\right) E_{s}-m\left(1-r_{\infty}\right) H_{c} \\
\frac{\mathrm{d}}{L \mathrm{~d} x} F_{2} & =-m F_{2}+\left(r_{\infty} s^{\prime}+s\right) E_{s}+m\left(1-r_{\infty}\right) H_{c} .
\end{aligned}
$$

Numerically stable analytical solutions of these differential equations are given by

$$
\begin{aligned}
F_{1} & =\delta_{1} e^{m L x}+\left(s^{\prime}+r_{\infty} s\right) E_{S}(0) \frac{e^{m L x}-e^{k L x}}{k-m}+\left(1-r_{\infty}\right) H_{c} \\
F_{2} & =\delta_{2} e^{-m L(1+x)} \\
& +\left(r_{\infty} s^{\prime}+s\right) E_{S}(0) \frac{e^{k L(1+x)}-e^{-m L(1+x)}}{k+m} e^{-k L}
\end{aligned}
$$




$$
+\left(1-r_{\infty}\right) H_{c}
$$

where $\delta_{1}$ and $\delta_{2}$ are constants which have to be determined from the boundary equations.

Defining the functions

$$
\begin{aligned}
& J_{1}(q, x)=\frac{e^{m L x}-e^{q L x}}{q-m} \\
& J_{2}(q, x)=\frac{e^{q L(1+x)}-e^{-m L(1+x)}}{q+m} e^{-q L},
\end{aligned}
$$

one can write

$$
\begin{aligned}
F_{1} & =\delta_{1} e^{m L x}+\left(s^{\prime}+r_{\infty} s\right) E_{s}(0) J_{1}(k, x)+\left(1-r_{\infty}\right) H_{c} \\
F_{2} & =\delta_{2} e^{-m L(1+x)}+\left(r_{\infty} s^{\prime}+s\right) E_{s}(0) J_{2}(k, x) \\
& +\left(1-r_{\infty}\right) H_{c} .
\end{aligned}
$$

Function $J_{2}(q, x)$ is numerically stable, but $J_{1}(q, x)$ must be approximated by a different function if $q-m$ is small, say less than $10^{-3}$. Thus we redefine

$$
\begin{aligned}
& J_{1}(q, x)= \\
& \begin{cases}\frac{e^{m L x}-e^{q L x}}{q-m} & |q-m| \geq 10^{-3} \\
\frac{-1}{2}\left(e^{m L x}+e^{q L x}\right) L x & \\
{\left[1-\frac{1}{12}(q-m)^{2} L^{2} x^{2}\right]} & |q-m|<10^{-3}\end{cases}
\end{aligned}
$$

The energy balance, and therefore also leaf temperatures, photosynthesis and fluorescence, depends on the direct and diffuse fluxes in the canopy. The direct solar flux follows directly from

$$
E_{S}(x)=E_{S}(0) P_{s}(x)
$$

where $P_{s}(x)=\exp (k L x)$ is the probability of sunshine (or the gap fraction in the direction of the sun), $L$ is the total LAI and $x$ the relative optical height, which runs from -1 at the bottom to zero at the canopy top.

The diffuse fluxes can be calculated once the transformed fluxes have been determined using Eq. (A8). However, this requires solving the boundary constants $\delta_{1}$ and $\delta_{2}$. This can be achieved by evaluating $F_{1}$ at the canopy top, and $F_{2}$ at the canopy bottom, giving

$$
\begin{gathered}
F_{1}(0)=\delta_{1}+\left(1-r_{\infty}\right) H_{c} \\
F_{2}(-1)=\delta_{2}+\left(1-r_{\infty}\right) H_{c} .
\end{gathered}
$$

Here, use was made of the fact that $J_{1}(k, 0)=0$, and also $J_{2}(k,-1)=0$. In terms of the normal diffuse fluxes one then obtains

$$
\begin{aligned}
\delta_{1} & =F_{1}(0)-\left(1-r_{\infty}\right) H_{c} \\
& =E^{-}(0)-r_{\infty} E^{+}(0)-\left(1-r_{\infty}\right) H_{c} \\
\delta_{2} & =F_{2}(-1)-\left(1-r_{\infty}\right) H_{c} \\
& =-r_{\infty} E^{-}(-1)+E^{+}(-1)-\left(1-r_{\infty}\right) H_{c} .
\end{aligned}
$$

If thermal emission is disregarded for the moment, the constants are given by

$\delta_{1}=E^{-}(0)-r_{\infty} E^{+}(0)$

$\delta_{2}=E^{+}(-1)-r_{\infty} E^{-}(-1)$.

Four-stream radiative transfer for the canopy-soil system can now be described by

$$
\begin{aligned}
E_{s}(-1) & =\tau_{s s} E_{s}(0) \\
E^{-}(-1) & =\tau_{s d} E_{s}(0)+\tau_{d d} E^{-}(0)+\rho_{d d} E^{+}(-1) \\
E^{+}(0) & =\rho_{s d} E_{s}(0)+\rho_{d d} E^{-}(0)+\tau_{d d} E^{+}(-1) \\
E^{+}(-1) & =r_{s}\left[E_{s}(-1)+E^{-}(-1)\right] .
\end{aligned}
$$

Here, the double-subscripted intrinsic reflectance and transmittance quantities of the isolated canopy layer are provided as output quantities of the 4SAIL model and are given by

$$
\begin{aligned}
\tau_{s s}= & e^{-k L} \\
\tau_{d d}= & \frac{\left(1-r_{\infty}^{2}\right) e^{-m L}}{1-r_{\infty}^{2} e^{-2 m L}} \\
\rho_{d d}= & \frac{r_{\infty}\left(1-e^{-2 m L}\right)}{1-r_{\infty}^{2} e^{-2 m L}} \\
\tau_{s d}= & \\
& \frac{\left(s^{\prime}+r_{\infty} s\right) J_{1}(k,-1)-r_{\infty} e^{-m L}\left(r_{\infty} s^{\prime}+s\right) J_{2}(k, 0)}{1-r_{\infty}^{2} e^{-2 m L}} \\
\rho_{s d}= & \frac{-r_{\infty} e^{-m L}\left(s^{\prime}+r_{\infty} s\right) J_{1}(k,-1)+\left(r_{\infty} s^{\prime}+s\right) J_{2}(k, 0)}{1-r_{\infty}^{2} e^{-2 m L}} .
\end{aligned}
$$

Combining the second and fourth equation of Eq. (A15) gives

$$
\begin{aligned}
& E^{-}(-1)-\rho_{d d} E^{+}(-1)=\tau_{s d} E_{s}(0)+\tau_{d d} E^{-}(0) \\
& -r_{s} E^{-}(-1)+E^{+}(-1)=r_{s} E_{s}(-1)=r_{s} \tau_{s s} E_{s}(0) .
\end{aligned}
$$

Solving $E^{+}(0)$ from these gives

$E^{+}(-1)=\frac{\left(\tau_{s d}+\tau_{s s}\right) E_{s}(0)+\tau_{d d} E^{-}(0)}{1-r_{s} \rho_{d d}} r_{s}$.

In the program FluorSAIL (Miller et al., 2005), a predecessor of SCOPE, the following additional equations are used to determine the boundary constants:

$$
\begin{aligned}
E^{+}(0) & =\rho_{s d} E_{S}(0)+\rho_{d d} E^{-}(0)+\tau_{d d} E^{+}(-1) \\
E^{-}(-1) & =\tau_{s d} E_{s}(0)+\tau_{d d} E^{-}(0)+\rho_{d d} E^{+}(-1) .
\end{aligned}
$$


Note, that only $E_{\mathrm{sun}}=E_{S}(0)$ and $E_{\mathrm{sky}}=E^{-}(0)$ are required as inputs for these calculations, since all other intrinsic canopy optical properties are provided by 4SAIL. Calculation of the internal diffuse fluxes in the canopy can now proceed by using Eq. (A8) and applying the inverse transformation to $F_{1}$ and $F_{2}$ to obtain back the original diffuse fluxes:

$$
\begin{aligned}
F_{1}(x) & =\delta_{1} e^{m L x}+\left(s^{\prime}+r_{\infty} s\right) E_{S}(0) J_{1}(k, x) \\
F_{2}(x) & =\delta_{2} e^{-m L(1+x)}+\left(r_{\infty} s^{\prime}+s\right) E_{s}(0) J_{2}(k, x) \\
E^{-}(x) & =\left[F_{1}(x)+r_{\infty} F_{2}(x)\right] /\left(1-r_{\infty}^{2}\right) \\
E^{+}(x) & =\left[r_{\infty} F_{1}(x)+F_{2}(x)\right] /\left(1-r_{\infty}^{2}\right) .
\end{aligned}
$$

\section{Appendix B}

\section{Aerodynamic resistance}

For aerodynamic resistance, the schematisation of Wallace and Verhoef (2000) was used. A two-source model was used with separate resistances for soil and canopy (Fig. B1).

Aerodynamic resistance in the inertial sublayer is:

$r_{a}^{I}=\frac{1}{K_{r} u_{*}}\left[\ln \left(\frac{z_{r}-d}{z_{R}-d}\right)\right]-\Psi_{h, v}\left(z_{r}\right)+\Psi_{h, v}\left(z_{R}\right)$,

where $K_{r}=0.41$ is Von Kármán's constant (the symbols $K$ an $\kappa$ are more common in the literature, but these are already reserved for extinction coefficients in this paper), $u_{*}$ is the friction velocity $\left(\mathrm{m} \mathrm{s}^{-1}\right), z_{r}$ the reference height $(\mathrm{m}), z_{R}$ height of the roughness sublayer (m), $d$ is the zero-plane displacement (m), and $\Psi_{h, v}$ a stability correction function (see below).

The aerodynamic resistance in the roughness sublayer is:

$r_{a}^{R}=\frac{z_{R}-h}{K_{r} u_{*}\left(z_{R}-d\right)}-\Psi_{h, v}^{*}\left(z_{R}\right)+\Psi_{h, v}^{*}(h)$,

where $h$ is the vegetation height, and $\Psi_{h, v}^{*}$ a stability correction function (see below). Aerodynamic resistance in the canopy, above the in-canopy source height $z_{0 \mathrm{~m}}+d$, is:

$r_{a}^{c}=\frac{h \sinh (n)}{n K_{h v}(h)}\left[\ln \left(\frac{e^{n}-1}{e^{n}+1}\right)-\ln \left(\frac{e^{n\left(z_{0 \mathrm{~m}}+d\right) / h}-1}{e^{n\left(z_{0 \mathrm{~m}}+d\right) / h}+1}\right)\right]$,

where $z_{0 \mathrm{~m}}$ is the roughness height for momentum (m), $n$ a wind extinction coefficient (see below), and $K_{h v}$ the eddy diffusivity (see below).

For the boundary layer resistance of leaves:

$r_{b}^{c}=\frac{70}{L} \sqrt{\frac{w_{l}}{u_{z 0 \mathrm{~m}}}}$,

where $w_{l}$ is leaf width (m) and $u_{z 0 \mathrm{~m}}$ wind speed at $z=z_{0 \mathrm{~m}}+\mathrm{d}$ (see below).

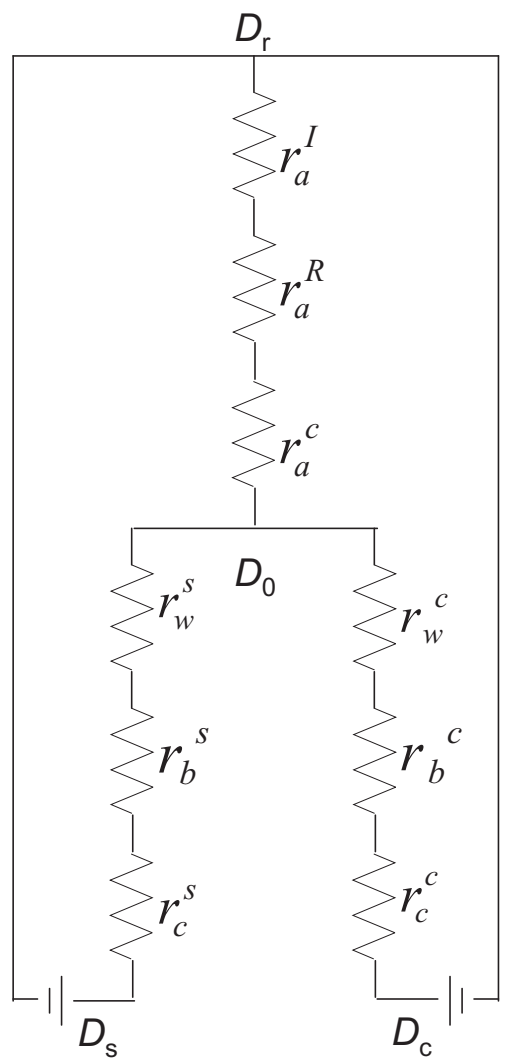

Fig. B1. Schematic representation of a two-source resistance model for resistances to water vapour and heat transport in and above the canopy, after Wallace and Verhoef (2000). Letter " $D$ " refers to either temperature $(T)$ or absolute humidity $(q)$. Other symbols are defined in Table 1.

The within canopy resistance is:

$r_{w}^{c}=$

$\frac{h \sin h(n)}{n K_{h v}\left(h_{1}\right)}\left[\ln \left(\frac{e^{n\left(z_{0 \mathrm{~m}}+d\right) / h}-1}{e^{n\left(z_{0 \mathrm{~m}}+d\right) / h}+1}\right)-\ln \left(\frac{e^{n \cdot 0.01 / h}-1}{\mathrm{e}^{n \cdot 0.01 / h}+1}\right)\right]$.

Here, the value of 0.01 denotes the roughness length of soil.

For the boundary layer resistance of soil and the surface resistance of vapour transport in soil pores, a priori values are used:

$r_{b}^{s}=150 \mathrm{sm}^{-1}$ and $r_{s}^{s}=500 \mathrm{sm}^{-1}$

The value for $r_{s}^{s}$ could be a function of soil moisture content (e.g. Buckley, 2005). For heat transport, $r_{s}^{c}=r_{s}^{s}=0$.

In the above equations, friction velocity $u_{*}\left(\mathrm{~m} \mathrm{~s}^{-1}\right)$ is:

$u_{*}=K_{r} u(z)\left[\ln \left(\frac{z-d}{z_{0 \mathrm{~m}}}\right)-\Psi_{m}\left(\frac{z-d}{\Lambda}\right)\right]^{-1}$,

where $\Lambda$ Monin-Obukhov length (m). Wind speed at height $z_{0 \mathrm{~m}}+\mathrm{d}, u_{z 0}$, is:

$u_{z 0}=u_{z} e^{n\left(\left(z_{0 \mathrm{~m}}+d\right) /(h-1)\right)}$. 
Wind extinction coefficient $n$ is calculated as:

$n=\frac{c_{d} L}{2 K_{r}^{2}}$,

where $c_{d}(=0.2)$ is a drag coefficient. The eddy diffusivity $K_{h, v}$ is calculated as:

$K_{h, v}=K_{r} u_{*}(z-d) \Phi_{m, h, v}^{-1}$,

where $z=z_{r}$ (see Eq. B1) or $z_{R}$ (Eqs. B1 and B2) or $h$ (Eq. B2).

The stability correction functions for neutral conditions are:

$\Psi_{h, v}=\Psi_{h, v}^{*}=\Psi_{m}=0$.

$\Phi_{m, h, v}^{-1}=1$

In the model, these values are also used for stable conditions (i.e. no stability correction is applied for stable conditions). For unstable conditions:

$\Psi_{h, v}=2 \log \left(\frac{1+x_{z}^{2}}{2}\right)$.

$\Psi_{h, v}^{*}=\frac{z-d}{z_{R}-d} \frac{x_{z}^{2}-1}{x_{z}^{2}+1}$.

$\Psi_{m}=2 \log \left(\frac{1+x_{z}}{2}\right)+\log \left(\frac{1+x_{z}^{2}}{2}\right)-2 \arctan \left(x_{z}\right)+\frac{\pi}{2}$.

$\Phi_{m, h, v}^{-1}=\left(1-16 \frac{h-d}{\Lambda}\right)^{1 / 2}$

In these equations:

$x_{z}=\left(1-16 \frac{z}{\Lambda}\right)^{1 / 4}$

Acknowledgements. This research was funded by The Netherlands Organisation for Scientific Research (NWO), SRON project number EO-071, and partly by the study "Impact assessment of solar induced vegetation fluorescence observations from space for improving dynamic vegetation models" for the European Space Agency under ESTEC Contract No. 20678/07/NL/HE. The authors thank Jérôme Demarty, Albert Olioso, Federico Magnani, Antonio Volta, Uwe Rascher and Alexander Damm for fruitful discussions and participation in modelling experiments, and Jan Elbers, Wim Timmermans, Remco Dost, Li Jia, Zoltán Vekerdy and Kitsiri Weligepolage for fieldwork, data processing and coordination during the SPARC and EAGLE campaigns. The authors also thank the reviewers for thoroughly reading the paper and providing useful suggestions.

Edited by: G. Wohlfahrt

\section{References}

Anderson, M. C., Norman, J. M., Kustas, W. P., Houborg, R., Starks, P. J., and Agam, N.: A thermal-based remote sensing technique for routine mapping of land-surface carbon, water and energy fluxes from field to regional scales, Remote Sens. Environ., 112, 4227-4241, doi:10.1016/j.rse.2008.07.009, 2008.

Baldocchi, D. D.: Assessing the eddy covariance technique for evaluating carbon dioxide exchange rates of ecosystems: past, present and future, Glob. Change Biol., 9, 479-492, doi:10.1046/j.1365-2486.2003.00629.x, 2003.

Bastiaanssen, W. G. M., Menenti, M., Feddes, R. A., and Holtslag, A. A. M.: A remote sensing surface energy balance algorithm for land (SEBAL). 1. Formulation, J. Hydrol., 212-213, 198-212, doi:10.1016/S0022-1694(98)00253-4, 1998.

Berk, A., Anderson, G. P., Acharya, P. K., Chetwynd, J. H., Bernstein, L. S., Shettle, E. P., Matthew, M. W., and Adler-Golden, S. M.: MODTRAN4 USER'S MANUAL, Air Force Research Laboratory, Space Vehicles Directorate, Air Force Materiel Command, Hanscom AFB, MA 01731-3010, 97 pp., 2000.

Bhumralkar, C. M.: Numerical Experiments on the Computation of Ground Surface Temperature in an Atmospheric General Circulation Model, J. Appl. Meteorol., 14, 1246-1258, 1975.

Buckley, T. N.: The control of stomata by water balance, New Phytol., 168, 275-292, 2005

Buschmann, C.: Variability and application of the chlorophyll fluorescence emission ratio red/far-red of leaves, Photosynth. Res., 92, 261-271, doi:10.1007/s11120-007-9187-8, 2007

Carter, G. A. and Knapp, A. K.: Leaf optical properties in higher plants: linking spectral characteristics to stress and chlorophyll concentration, Am. J. Bot., 88, 677-684, 2001.

CEOS: The Earth Observation Handbook - Climate change special edition 2008 (ESA SP-1315), edited by: Bond, P., ESA Communications Production Office, Noordwijk, The Netherlands, 2008.

Collatz, G., Ribas-Carbo, M., and Berry, J. A.: Coupled photosynthesis-stomatal conductance model for leaves of $\mathrm{C} 4$ plants, Aus. J. Plant Physiol., 19, 519-538, 1992.

Cowan, I.: Stomatal behaviour and environment, Adv. Bot. Res., 4, 117-228, 1977.

Entcheva Campbell, P. K., Middleton, E. M., Corp, L. A., and Kim, M. S.: Contribution of chlorophyll fluorescence to the apparent vegetation reflectance, Sci. Total Environ., 404, 433-439, doi:10.1016/j.scitotenv.2007.11.004, 2008.

Farquhar, G., Von Caemmerer, S., and Berry, J.: A biochemical model of photosynthetic $\mathrm{CO} 2$ assimilation in leaves of $\mathrm{C} 3$ species, Planta, 149, 78-90, 1980.

Genty, B., Birantais, J., and Baker, N.: The relationship between the quantum efficiencies of photosystems I and II in pea leaves, Biochem. Biophys. Acta, 990, 87-92, 1989.

Goudriaan, J.: Crop micrometerology: a simulation study, PUDOC, Wageningen, 249 pp., 1977.

Gu, L., Baldocchi, D., Verma, S. B., Black, T. A., Vesela, T., Falge, E. M., and Dowty, P. R.: Advantages of diffuse radiation for terrestrial ecosystem productivity, J. Geophys. Res., 107(D6), 4050, doi:10.1029/2001JD001242, 2002.

Guilioni, L., Jones, H. G., Leinonen, I., and Lhomme, J.-P.: On the relationships between stomatal resistance and leaf temperatures in thermography, Agr. Forest Meteorol., 148, 1908-1912, doi:10.1016/j.agrformet.2008.07.009, 2008.

Hall, F. G., Huemmrich, K. F., Goetz, S. J., Sellers, P. J., and 
Nickeson, J. E.: Satellite remote sensing of surface energy balance: success, failures, and unresolved issues in FIFE, J. Geophys. Res., 97(D17), 19061-19089, 1992.

Houldcroft, C.: Measuring and modeling the surface temperature and structure of a maize canopy, Ph.D. thesis, The University of Reading, UK, 235 pp., 2004.

Jacquemoud, S. and Baret, F.: PROSPECT: A model of leaf optical properties spectra, Remote Sens. Environ., 34, 75-91, 1990.

Jacquemoud, S., Bacour, C., Poilvé, H., and Frangi, J.-P.: Comparison of four radiative transfer models to simulate plant canopies reflectance: direct and inverse mode, Remote Sens. Environ., 74, 471-481, 2000.

Kull, O. and Kruyt, B.: Acclimation of photosynthesis to light: a mechanistic approach, Funct. Ecol., 13(1), 24-36, 1998.

Kustas, W. P., Anderson, M. C., French, A. N., and Vickers, D.: Using a remote sensing field experiment to investigate fluxfootprint relations and flux sampling distributions for tower and aircraft-based observations, Adv. Water Resour., 29, 355-368, doi:10.1016/j.advwatres.2005.05.003, 2006.

Kustas, W. P., Anderson, M. C., Norman, J. M., and Li, F.: Utility of radiometric-aerodynamic temperature relations for heat flux estimation, Bound.-Lay. Meteorol., 122, 167-187, 2007.

Lloyd, J. and Farquhar, G.: $13 \mathrm{C}$ discrimination during $\mathrm{CO}_{2}$ assimilation by the terrestrial biosphere, Oecologia, 99, 201-215, 1994.

Mäkelä, A., Hari, P., Berninger, F., Hänninen, H., and Nikinmaa, E.: Acclimation of photosyn-thetic capacity in Scots pine to the annual cycle of temperature, Tree Physiol., 24, 369-376, 2004.

Miller, J., Berger, M., Goulas, Y., Jacquemoud, S., Louis, J., Mohammed, G., Moise, N., Moreno, J., Moya, I., Pedrós, R., Verhoef, W., and Zarco-Tejada, P.: Development of a Vegetation Fluorescence Canopy Model, ESTEC Contract No. 16365/02/NL/FF, Final Report, 138 pp., 2005.

Nikolov, N. T., Massman, W. J., and Schoettle, A. W.: Coupling biochemical and biophysical processes at the leaf level: an equilibrium photosynthesis model for leaves of C3 plants, Ecol. Model., 80, 205-235, doi:10.1016/0304-3800(94)00072-P, 1995.

Norman, J. M.: Modeling the complete crop canopy, in: Modification of the aerial environment of plants, edited by: Barfield, B. J. and Gerber, J. F., ASAE Monogr. Am. Soc. Agric. Engr., St. Joseph, MI, 249-277, 1979.

Norman, J. N. and Becker, F.: Terminology in thermal infrared remote sensing of natural surfaces, Agr. Forest Meteorol., 77, 153166, doi:10.1016/0168-1923(95)02259-Z, 1995.

Rascher, U., Gioli, B., and Miglietta, F.: FLEX - Fluorescence Explorer: A Remote Sensing Approach to Quantify SpatioTemporal Variations of Photosynthetic Efficiency from Space, in: "Photosynthesis. Energy from the Sun, 14th International Congress on Photosynthesis", edited by: Allen, J. F., Gantt, E., Golbeck, J. H., and Osmond, B., Springer, Netherlands, 2008.

Reich, P. B., Ellsworth, D. S., Walters, M. B., Vose, J. M., Gresham, C., Volin, J. C., and Bowman, W. D.: Generality of leaf trait relationships: a test across six biomes, Ecology, 80, 1955-1969, 1999.

Sellers, P. J., Dickinson, R. E., Randall, D. A., Betts, A., Hall, F., Berry, J., Collatz, G., Denning, A., Mooney, H., Nobre, C., Sato, N., Field, C., and Henderson-Sellers, A.: Modeling the exchanges of energy, water, and carbon between continents and the atmosphere, Science, 275(5299), 502-509, 1997.

Smolander, H. and Stenberg, P.: A method to account for shoot scale clumping in coniferous canopy reflectance models, Remote Sens. Environ., 88, 363-373, doi:10.1016/j.rse.2003.06.003, 2003.

Su, Z.: The Surface Energy Balance System (SEBS) for estimation of turbulent heat fluxes, Hydrol. Earth Syst. Sci., 6, 85-100, 2002, http://www.hydrol-earth-syst-sci.net/6/85/2002/.

Su, Z., Timmermans, W. J., Gieske, A., Jia, L., Elbers, J. A., Olioso, A., Timmermans, J., Van der Velde, R., Jin, X., Van der Kwast, H., Nerry, F., Sabol, D., Sobrino, J. A., Moreno, J., and Bianchi, R.: Quantification of land-atmosphere exchanges of water, energy and carbon dioxide in space and time over the heterogeneous Barrax site, Int. J. Remote Sens., 29(17), 5215-5235, 2008.

Su, Z., Timmermans, W. J., van der Tol, C., Dost, R., Bianchi, R., Gómez, J. A., House, A., Hajnsek, I., Menenti, M., Magliulo, V., Esposito, M., Haarbrink, R., Bosveld, F., Rothe, R., Baltink, H. K., Vekerdy, Z., Sobrino, J. A., Timmermans, J., van Laake, P., Salama, S., van der Kwast, H., Claassen, E., Stolk, A., Jia, L., Moors, E., Hartogensis, O., and Gillespie, A.: EAGLE 2006 Multi-purpose, multi-angle and multi-sensor in-situ and airborne campaigns over grassland and forest, Hydrol. Earth Syst. Sci., 13, 833-845, 2009,

http://www.hydrol-earth-syst-sci.net/13/833/2009/.

Timmermans, J., Van der Tol, C., Verhoef, W., and Su, Z.: Contact and directional radiative temperature measurements of sunlit and shaded land surface components during the SEN2FLEX 2005 campaign, Int. J. Remote Sens., 29(17-18), 5183-5192, 2008.

Tuzet, A., Perrier, A., and Leuning, R.: A coupled model of stomatal conductance, photo-synthesis and transpiration, Plant Cell Environ., 26, 1097-1116, 2003.

Van der Tol, C., Verhoef, W., and Rosema, A.: A model for chlorophyll fluorescence and photosynthesis at leaf scale, Agr. Forest Meteorol., 149(1), 96-105, 2009.

Verhoef, W.: Light scattering by leaves with application to canopy reflectance modelling: the SAIL model, Remote Sens. Environ., 16, 125-178, 1984.

Verhoef, W.: Theory of radiative transfer models applied in optical remote sensing of vegetation canopies, Ph.D. thesis, Wageningen Agricultural University, 1998.

Verhoef, A. and Allen, S. J.: A SVAT scheme describing energy and $\mathrm{CO}_{2}$ fluxes for multi-component vegetation: calibration and test for a Sahelian savannah, Ecol Model., 127, 245-267, 2000.

Verhoef, W. and Bach, H.: Remote sensing data assimilation using coupled radiative transfer models, Phys. Chem. Earth Pt. A/B/C, 28, 3-13, 2003.

Verhoef, W. and Bach, H.: Coupled soil-leaf-canopy and atmosphere radiative transfer modeling to simulate hyperspectral multi-angular surface reflectance and TOA radiance data, Remote Sens. Environ., 109(2), 166-182, 2007.

Verhoef, W., Jia, L., Xiao, Q., and Su, Z.: Unified optical-thermal four-stream radiative transfer theory for homogeneous vegetation canopies. IEEE T. Geosci. Remote, 45(6), 1808-1822, 2007.

Von Caemmerer, S. and Baker, N.: The biology of transpiration: from guard cells to globe, Plant Physiol., 143, 3 pp, 2007.

Wallace, J. S. and Verhoef, A.: Modelling interactions in mixedplant communities: light, water and carbon dioxide, in: Leaf Development and Canopy Growth, edited by: Marshall, B. and Roberts, J. A., Sheffield Academic Press, UK, 2000.

Wullschleger, S. D.: Biochemical limitations to carbon assimilation in $\mathrm{C} 3$ plants - A retrospective analysis of the $\mathrm{A} / \mathrm{Ci}$ curves from 109 species, J. Exp. Bot., 44, 907-920, 1993. 